\title{
Permeability of Laboratory-Formed Methane-Hydrate-Bearing Sand: Measurements and Observations Using X-ray Computed Tomography
}

Timothy J. Kneafsey/Lawrence Berkeley National Laboratory (LBNL), Yongkoo Seol/ National Energy Technology Laboratory, Arvind Gupta/Colorado School of Mines (now at Shell), Liviu Tomutsa, SPE/LBNL

\begin{abstract}
Methane hydrate was formed in two moist sands and a sand-silt mixture under a confining stress in an x-ray transparent pressure vessel. Three initial water saturations were used to form three different methane hydrate saturations in each medium. X-ray computed tomography (CT) was used to observe locationspecific density changes caused by hydrate formation and flowing water. Gas permeability measurements in each test for the dry, moist, frozen, and hydrate-bearing states are presented. As expected, the effective permeabilities (intrinsic permeability of the medium multiplied by the relative permeability) of the moist sands decreased with increasing moisture content. In a series of tests on a single sample, the effective permeability typically decreased as the pore space became more filled, in the order of dry, moist, frozen, and hydrate-bearing. In each test, water was flowed through the hydrate-bearing medium, and we observed the location specific changes in water saturation using CT scanning. We compared our data to a number of models, and our relative permeability data compare most favorably with models in which hydrate occupies the pore bodies rather than the pore throats. Inverse modeling (using the data collected from the tests) will be performed to extend the relative permeability measurements.
\end{abstract}

\subsection{Introduction}

Gas hydrates are crystalline assemblages of water that form cage-like spaces occupied by guest molecules such as methane or carbon dioxide. Naturally occurring gas-hydrate-bearing deposits present in the permafrost and in suboceanic environments have been identified as potential large sources of natural gas. The production of natural gas from hydrates will pose many challenges, among which is the economic viability of producing gas at a particular location. This interest in gas hydrates has induced many field, laboratory, and modeling studies focused on various aspects of gas hydrates in the natural environment.

Methane hydrate (herein after referred to as "hydrate"), thought to be the most predominant natural gas hydrate, is not stable at atmospheric conditions, requiring a combination of cold temperature and high gas pressure for stability (Figure 1)[Moridis et al., 2007]. For that reason, pristine samples of natural gas hydrates have been difficult to retrieve and make measurements on. Pressure coring has been successfully performed at a few locations retrieving the most representative samples so far; however, these are not readily available for all studies, or in some cases not amenable to the needed study.

Because of this difficulty, several techniques have been developed to make gas hydrates for laboratory study. In the ice-to-hydrate method [Stern et al., 1996], ice is melted in the presence of methane at the appropriate pressure forming hydrate. The hydrate can then be pulverized, mixed with a mineral substrate, and compacted to create laboratory samples of methane hydrate in a mineral medium, with the hydrate being part of the load-bearing frame of the medium. In the excess gas method [Handa and Stupin, 1992], water is distributed throughout a mineral substrate (e.g.. packed moist sand, drained sand, moistened silica gel, ...) and the mixture is brought to hydrate-stable conditions (chilled and pressurized with gas), allowing hydrate to form. It is thought that this method produces grain-cementing hydrate from the pendular water [Waite et al., 2004]. In the dissolved gas method [Tohidi et al., 2001], water containing sufficient dissolved guest molecules is brought to hydrate-stable conditions forming hydrate. In the laboratory, this is usually done by pre-dissolving the gas of interest in water and then introducing it to the sample under the appropriate hydrate-forming conditions, and it is easier to form hydrate from more soluble gases such as carbon dioxide. It is thought that this method more closely simulates the way most natural gas hydrate has formed. However, laboratory implementation of this method is difficult, and sample formation is 
prohibitively time consuming [Minagawa et al., 2005; Spangenberg and Kulenkampff, 2005].

Permeability (the resistance to fluid flow under a pressure gradient) and relative permeability (the effect of the presence of other phases such as gas hydrate, that interfere with the fluid flow of interest) are among the most important parameters governing gas production from a hydrate-bearing reservoir. From the gasproduction perspective, a large gas hydrate reserve with low permeability can be less desirable than a smaller reserve with higher permeability. Understanding how gas hydrate in the pore space affects permeability, and how it affects water and gas relative permeability is necessary to more accurately predict natural gas production and the economic viability of hydrate-bearing reservoirs.

Very few permeability and relative permeability measurements of hydrate-bearing sediments have been performed. Jaiswal [2004] measured the relative permeability of samples of two sands having different hydrate saturations. Prior to hydrate formation, the sands were drained in a controlled manner to provide different initial water saturations, and gas hydrate was formed using the excess gas method. The relative permeabilities inferred from these unsteady-state core floods were thought to encompass not only resistance to flow, but also the effects of dissociation instabilities caused by fluid flow, fine particle migration, and local compaction in porous media at low temperature, which are strongly impacted by hydrate distribution in the samples and within the pores. These results clearly indicate the need for multiple sets of permeability data for other sample types and methods to provide confidence in the parameters used in the models prior to the expenditure of large sums of money in the field.

Minagawa et al. [2005] measured the water permeability of several hydrate-bearing sands at various hydrate saturations. In these tests, hydrate was also formed using the excess gas method. An exponential relation $\left(k=k_{0}\left(1-S_{h}\right)^{n}\right.$-where $k$ is permeability with hydrate, $k_{0}$ is the permeability without hydrate, and $S_{h}$ is the hydrate saturation) - was used to fit the measured data. Even though the sands were fairly similar, the exponent $n$ values varied over a wide range (2.5 to 9.8). Kumar et al. [2010] measured gas permeability of carbon dioxide hydrate bearing glass beads and compared their results to a numerical model. These authors concluded that at hydrate saturations (formed using the excess gas method) below $35 \%$, hydrate formed as coatings on the grains, and at saturations above $35 \%$ and below $49 \%$, the hydrate was pore filling.

X-ray CT scanning (CT) has been applied to hydrate research for hydrate in porous media and for pure hydrate. Uchida et al. [2000a] used CT scanning to observe hydrate dissociation of natural hydrate-bearing samples. CT scanning was used by Gupta et al. [2006] and Kneafsey et al. [2007], to observe the distribution of hydrate formed in laboratory samples, in both pure hydrate and hydrate within a porous medium. Gupta and Kneafsey both observed that the hydrate distribution was affected by water migration caused by capillary pressure changes that occurred from forming the solid hydrate phase in the pore space. Because many physical properties of hydrate-bearing sediments - such as thermal conductivity [Waite et al., 2002], permeability [Jaiswal, 2004; this work], and acoustic wave speeds [Waite et al., 2004] —are dependant upon the hydrate saturation and location within the porespace, the configuration of the formed hydrate strongly affects the results of a test [Waite et al., 2008]. Using x-ray microtomography, Kerkar and coworkers [Kerkar et al., 2009] imaged the three dimensional growth of THF hydrate in bead packs. The resulting THF hydrate did not wet the mineral surfaces and geometrically resembled oil blobs in water-wet porous media.

We have performed a series of tests in which methane hydrate was formed in moist sand packs contained under a confining stress within an $\mathrm{x}$-ray transparent pressure vessel. Confining stress was applied to minimize mineral grain redistribution resulting from the freezing/thawing and hydrate formation/dissociation phase changes, and also to assure that no flow bypassed the sample during flow tests. As observed by Kneafsey et al. [2007], hydrate formation in a well-packed sand pack in a rigid container induced strong density changes in a sample, possibly creating a high-permeability pathway around the sample. For each sample, the gas permeability was measured for a series of conditions including moist, frozen, hydrate bearing, and dry. Additionally, we performed waterfloods in our samples to gather data on water flow and used CT to monitor water saturation changes at multiple locations. Using CT imaging, we are able to monitor spatially dependent processes and more correctly attribute them to their proper locations, rather than to the bulk sample as would occur if we were limited to pressure and temperature measurements alone. 


\subsection{Method}

Our test procedure was developed so that we performed a number of tests on the same sample to examine changes caused by the applied conditions. The initially moist sample was packed into the elastomer sleeve in an x-ray transparent pressure vessel, and a series of temperature and pressure conditions were applied. The typical test procedure is described below, with slight modifications from test to test:

1. Mix water and sand/silt to achieve the desired moisture content

2. Pack the moist sand/silt in thin lifts in the rubber sleeve

3. Apply confining pressure (manually adjusted to impose an effective stress [confining pressure less pore pressure])

4. Connect the flow system

5. Pressurize the sample with nitrogen and eliminate leaks

6. Measure the gas permeability of the moist sand using a small quantity of nitrogen at a low flow rate

7. CT scan the initially moist sand sample

8. Freeze the water in the sample

9. CT scan the frozen sample

10. Measure the gas permeability of the frozen sample

11. Thaw the ice resulting in a moist sand pack

12. Flush the nitrogen gas with methane

13. Create methane hydrate in the sample by increasing methane pressure while maintaining a cool temperature $\left(\sim 4^{\circ} \mathrm{C}\right)$ (excess gas method)

14. Measure the sample gas permeability using methane

15. CT scan the hydrate-bearing system

16. Perform a waterflood under hydrate-stable conditions while CT scanning

17. Perform a water permeability test at residual gas saturation

18. Dissociate the hydrate in the sample while CT scanning

19. Evacuate the sample, saturate it with water and measure single fluid phase permeability

20. CT scan the water-saturated system

21. Dry the sample in the sleeve by flushing with isopropyl alcohol, followed by dry nitrogen gas

22. CT scan the dried sample to allow saturation quantification

23. Perform a waterflood of the dry sand (i.e., a "dry” waterflood)

Tests were performed at three initial water saturations in each of three porous media. Initial conditions, resulting hydrate saturation, remaining gas saturation, and the amount of water converted to hydrate are listed in Table 1.

\subsection{Test Materials and Equipment}

Silica sand or sand/silt (Figure 2) was step-wise moistened and mixed until the desired moisture content was achieved. The sand was left to equilibrate in a sealed plastic bag for several hours and remixed prior to packing in the elastomer sleeve. The masses of the bag, sand, and water were recorded to allow calculation of moisture content and water saturation. To pack the elastomer sleeve, we assembled one end of the pressure vessel (Figure 3, top) and inserted a $5 \mathrm{~cm}$ diameter x $3.8 \mathrm{~cm}$ long Teflon plug to provide thermal insulation between the sample and the stainless steel end cap. A $0.6 \mathrm{~cm}$ hole through the center of the Teflon plug was packed with stainless steel mesh to restrain sand grains. For the sand/silt tests, we used a different pressure vessel (Figure 3 bottom) with one floating and one fixed PVC end piece within the pressure vessel.

The sand was tamped into the rubber sleeve from the outlet to the inlet end in approximately $1 \mathrm{~cm}$ thick lifts, using rods with diameters of $1.25 \mathrm{~cm}, 2.5 \mathrm{~cm}$, and $3.75 \mathrm{~cm}$ ( 30 blows per rod to each lift) to compact the sand. In the region near the thermocouple at the outlet end, a $0.6 \mathrm{~cm}$ steel tube was placed over the thermocouple to protect it from the packing rods during compaction. This tube was raised the equivalent of the lift thickness $(\sim 1 \mathrm{~cm})$ for each layer of sand added. The flexible rubber sleeve was not confined during packing, resulting in some bulging and a nonuniform diameter. Upon filling the sleeve to the appropriate level, we inserted a $5 \mathrm{~cm}$ diameter $\times 3.8 \mathrm{~cm}$ long Teflon plug and then the stainless steel end cap containing a thermocouple that extended about $1 \mathrm{~cm}$ into the sample. 
The pressure vessel used for the Fsand and Ksand samples (DCHR-2.0, Temco, Tulsa, OK), shown in the top of Figure 3, accommodates samples up to about $5 \mathrm{~cm}$ in diameter and up to $45 \mathrm{~cm}$ in length, and has five ports on the side for accessing the sample. (The ports were used in earlier tests, but their use was abandoned to minimize leaks. The ports contained steel fittings that created shadows and artifacts in about $20 \%$ of the CT images.) The vessel was fitted into a PVC jacket through which temperature-controlled water/propylene glycol mixture flowed. The entire system was surrounded with an insulating box and mounted on the CT scanner table to reduce table-positioning errors. For the sand/silt test, a smaller pressure vessel was used to accommodate samples $5 \mathrm{~cm}$ in diameter and up to $15 \mathrm{~cm}$ in length (Figure $3 \mathrm{bottom}$ ).

A modified Siemens HiQ medical x-ray computed tomography (CT) scanner was used to obtain CT images. Sequential $0.5 \mathrm{~cm}$ image slices were collected over the length of the samples. The voxel size for the CT images for this set of tests was $\sim 0.2 \times 0.2 \times 5 \mathrm{~mm}$. CT scans were performed at all steady-state conditions in addition to monitoring the transient waterfloods. An example of how the CT imaging was performed is shown in Figure 4. Data obtained from the CT scanner (numerical values) are converted to density using a calibration curve developed by scanning objects having known density. Using the calibrated density for each location under each condition (dry, water saturated, moist, frozen, hydrate bearing), physical properties of the sand, water, ice, hydrate, and gas, with mass balance allows us to attribute phase saturations to specific locations. These numerical data are plotted here in grayscale or color images.

Type-K thermocouples (Omega Engineering, Stamford CT) were used to measure temperature in the room, the temperature-controlled bath, locations within the sample near both ends, and the gas bottle containing the methane gas. Pressure was measured independently at each end of the sample and at the methane gas bottle using Ashcroft K1 pressure transducers (Stratford, CT). Differential pressure across the sample (and end caps) was measured using a Rosemount 1151 pressure transducer (Chanhassen, MN) optimized for 0 to $0.14 \mathrm{MPa}$.

\subsection{Gas Permeability Measurement}

Two ISCO 500D syringe pumps (Lincoln, NE) were used to control flow during permeability measurements. The downstream pump was set to provide constant fluid pressure, typically around $5 \mathrm{MPa}$. The upstream pump was set to provide a series of constant flow rates $(0.5$ to $50 \mathrm{~mL} / \mathrm{min})$. Each flow rate was imposed and maintained until the system achieved a steady state, defined as both inlet and outlet pumps flowing at the same rate and the pressure differential across the sample reaching a fairly constant value for more than 60 seconds (four consecutive similar readings at 20-second intervals). At least four flow rates were used in each permeability measurement. Permeability was computed for each flow rate, and the average and standard deviation of these computed permeabilities are presented here. Typically, less than $500 \mathrm{~mL}$ of gas was flowed during a permeability measurement (including all the flow rates), thus minimizing drying of the sample during this step.

\subsection{Hydrate Formation}

Hydrate was formed in the samples by maintaining the temperature near $4^{\circ} \mathrm{C}$ and increasing the methane gas pressure to within the hydrate stability region (typically 5 to $5.5 \mathrm{MPa}$ ). During hydrate formation, the sample was connected to a methane reservoir having a known volume, temperature, and pressure. Quantification of the amount of hydrate formed was performed by calculating the number of moles of methane removed from the gas phase (from the pressure change), using appropriate volumes, pressures, and temperatures and methane properties from Lemmon et al. [2005].

\subsection{Waterfloods}

Waterfloods were conducted on samples when hydrate was present, and again in some samples when the sample was dry. For these waterfloods, water was flowed at a constant rate (on the order of $1 \mathrm{~mL} / \mathrm{min}$ ) into the sample. Water saturation and distribution were monitored at several locations (cross-sectional slices) using CT. Waterfloods through hydrate-bearing samples were performed to provide visualization of water flowing through hydrate-bearing media, and to collect water saturation data at specific locations. These data are being used for estimating the relative permeability of water and gas in hydrate-bearing medium by numerical inversion. Waterfloods in the dry samples were performed to allow for computation of parameters for the dry sand. 


\subsection{Results \\ 3.1 Hydrate Formation}

In all samples, hydrate formed throughout the sample, but in most, it tended to form more preferentially in the center of the sample. The hydrate distribution throughout each sample varied some from location to location. Figure 5 shows a single cross section from each of the nine hydrate-bearing samples (three media and three initial water saturations) that illustrates the "characteristic" hydrate formation patterns in the each sample. The hydrate saturation distribution at each voxel in the sample computed from CT, pressure, and temperature data are summarized in Figure 6. Most samples show somewhat broad hydrate saturation distributions, indicating that the hydrate saturation in the samples was not uniform across the samples. A few show multimodal hydrate saturation distributions (e.g., FSandSilt56) indicating the presence of regions with higher (or lower) varying hydrate saturations. One sample (Fsand40) shows multimodality and wide distribution across the saturation range. These distributions are consistent with the characteristic hydrate saturations shown in Figure 5.

\subsection{Gas Permeability}

Gas permeabilities measured in these tests are presented in Figure 7 as a function of the gas saturation. For the moist case and the frozen case, there are only two phases (water or ice, and gas) in the porespace. Because hydrate formation did not generally consume $100 \%$ of the water, the hydrate-bearing samples contained three phases (gas, hydrate, and unconverted water). In all cases, the permeability of the icebearing sand was less than the moist sand. This is as expected, because the water will expand when freezing further blocking the porespace available to gas flow. When hydrate forms from a fixed amount of water, the expansion is even greater than ice formation because methane is incorporated into the structure, adding mass to the resulting crystal, even though the density of methane hydrate and ice are approximately the same. The formation of hydrate in the porespace typically reduced the gas permeability for the moderate and higher initial water saturations, but in one case (Ksand 20), the gas permeability increased from the moist case upon hydrate formation.

Figure 8 presents the gas relative permeabilities (measured effective permeabilities divided by the intrinsic permeability) for the nine tests plotted against the gas saturation in the sample. The intrinsic gas permeability for the FsandSilt samples could not be directly measured because fines migration occurred during the waterflood modified the samples. The intrinsic permeability for these samples was instead computed using the Millington-Quirk equation [Moldrup et al., 1998] based on the gas permeabilities for the three moist samples. The spread in the data is noteworthy; however, several trends can be observed. First, the relative change in gas permeability with the different phases (water-ice-hydrate) is different for each medium. This is observable by following the trend of similar-sized, similar-color symbols. For the three media at the highest gas saturations (lowest initial water saturations), permeability does not necessarily follow the order moist $>$ frozen $>$ hydrate bearing as would be expected. This is because water migrates during freezing and hydrate formation causing regions of low and high effective permeability. Because of the cylindrical geometry of the sample and radial heat transfer, these regions tend to be somewhat contiguous throughout the sample. Although this behavior also happens at higher initial water saturations (lower gas saturations), the change in permeability does proceed in the order moist>frozen>hydrate bearing. Secondly, the relative magnitude of the permeability change is different for each medium. For the Ksand, the relative change is the greatest, followed by the mixed sand/silt, and the Fsand.

After hydrate dissociation for each medium, water was flowed through the sample until only residual gas remained. The water permeability with residual gas is presented for Ksand in Figure 7. No hydrate was present in this case. The average residual gas saturation across the sample computed using CT data was $28 \%$ (ranging from $20 \%$ to $31 \%$ ), with lower gas saturation near the inlet.

\subsection{Waterfloods}

Waterfloods were performed for the Fsand (dry, Fsand28 with hydrate, Fsand40 with hydrate), the Ksand (dry, Ksand 20 with hydrate, Ksand 28 with hydrate), and FsandSilt (11\% water saturation, FsandSilt23 with hydrate, FsandSilt39 with hydrate, and FsandSilt56 with hydrate). Waterfloods were not possible for the highest hydrate-saturated conditions for the Fsand56 and Ksand42 because the permeability was too 
low. During waterfloods, we repeatedly CT-scanned the sample at several specific cross-sectional locations to monitor water saturation over time (e.g., Figures 9, 11, and 12 show scans at a single location over time). Data acquired during each waterflood include pressure, pressure differential, and temperature near the two ends of the sample.

Figure 9a shows water saturation during a waterflood at one cross section (Loc 75, which is $65 \mathrm{~mm}$ from the fluid inlet - see Figure 3, top) for the dry Ksand. The figure shows sequential images from left to right and top to bottom that describe the saturation evolution for the same location over time. Water first appears in the fourth slice at the bottom. As time progressed, the bottom of the slice became satiated (but not saturated, owing to the presence of residual gas), and the water gradually satiated the slice. The impact of gravity is clearly visible: the water does not appear everywhere in the slice at once, but rather the slice fills from bottom to top. Note that while these scans provide two-dimensional images of a single slice which is part of a three-dimensional system, with flow coming into this slice from the adjacent slice (not shown) and going into the slice on the other side (also not shown).

Figure 9 b shows the hydrate saturation distribution at Location 75 for Ksand20, and Figure 9 c shows the water saturation at Location 75 during the waterflood with hydrate present over about 3 hours. The initial water and hydrate saturations at this location were calculated by taking the density change from the dry condition from CT data, and partitioning it between hydrate and water in proportion to the amount of hydrate formed. We assumed that the ratio of hydrate to remaining water is constant everywhere in the sample to allow this partitioning. This assumption might be verifiable using dual-energy CT scanning or other techniques; however, these were not performed here. The initial water saturation based on these assumptions is highest in the center (as was the hydrate saturation), and water was present throughout the sample.

As with the dry case, during the waterflood water initially reached this location at the sample bottom, indicating again the importance of gravity for this medium. As time progressed, the flowing water began to satiate the bottom, and the wet zone extended upwards, initially going around the center region that has higher hydrate saturation (Figure $9 \mathrm{~b}$ ). At the beginning of the third row, water began to imbibe into the center, while the wet zone continued to move upward outside of the center. The water saturation at the bottom began to get very high (about the same as the dry waterflood in spite of the hydrate presence in the porespace), the region outside of the center became highly saturated, and then finally water imbibed into the center, resulting in a somewhat uniformly saturated sample. Figure 10 shows saturation changes in three regions in this slice. When the water front reached the higher- $S_{\mathrm{h}}$ center, some water quickly imbibed there, but after that, the $S_{w}$ at the center increased only slowly. In the meantime, the $S_{w}$ at the top increased rapidly and leveled off before $S_{w}$ at the center reached its maximum.

Figure 11a shows water saturation at Loc 125 during the waterflood for the Fsand under initially dry conditions. In contrast to the slightly coarser Ksand, water initially appears just below the sample center, indicating the importance of capillary strength, gravity, and heterogeneity. In a water-wetting dry porous medium with a fairly uniform pore-size distribution, water will preferentially imbibe into more tightly packed regions with smaller pore sizes (higher capillary strength). In packing the sand in the rubber sleeve, it is difficult to pack the outer regions as well as the center because of radius differences between the tamping rod and the sleeve, and also because the sleeve expands when each tamping blow is delivered, and relaxes between blows. This forms a region at the outer radii of the sample with a slightly higher porosity, and consequently slightly larger pore sizes in that region in spite of the apparent homogeneity. The region of increasing water saturation in the center grows over time, and about halfway through the third row, the effect of gravity becomes more noticeable as the increases in saturation occur from bottom to top, but in a much more diffused way than for the slightly coarser Ksand.

Figure 11b shows the fairly uniform hydrate saturation at Loc 125 for the Fsand28 sample. In the water flood of the Fsand hydrate-bearing sample shown in Figure 11c, capillarity is clearly dominant since the water saturation increases initially in the top-right quadrant within this initially fairly uniformly partially hydrate-saturated sample. Unlike the dry case in Figure 11a, the local water saturation rapidly reaches its maximum and stays there, with the size of the wetted region growing over time. The impact of the less dense region at the outer radii of the slice is apparent in this test as well, since this is the region that satiates 
last.

Figure 12a shows the water saturation at Loc 295 in the initially moist (about 11\% initial water saturation) FsandSilt sample. Water reached the slice at the bottom of the sample in a somewhat diffuse manner in the sixth image, and the wetted zone increased vertically upward, going around the sample center. In the eighth image, water imbibition into the center had begun. By the first image in the second row, the saturation at the outer fringe of the center region had increased considerably; after which the saturation in the center continued to increase. Packing may be responsible for this behavior: higher porosity and permeability in the outer region from packing. As discussed above, the packing in the outer region is thought to be slightly less dense than the center. While packing, we noticed that the addition of silt to the sand seemed to make the mix flow better as if the smaller silt particles "lubricated" the sand grains. This would have the effect of making the higher-porosity region on the outside of the sample larger in size because the sand grains would be less able to lock onto each other.

Figure 12b shows the hydrate saturation for the FsandSilt 39 sample at Location 340. Figure 12c shows water saturations during the waterflood through the hydrate-bearing FsandSilt39 sample at Loc 340. As seen in Figure 12b, the hydrate saturation is higher in the center of the sample, and decreases towards the edges. Water flow in this slice begins in the outer reaches, with water saturation increasing towards the center over time. There is no two-region type behavior like in the nonhydrate-bearing waterflood, indicating that the presence of hydrate has a larger effect than packing artifacts. It would also be expected that the hydrate would inhibit fines migration by holding it in place.

There are three dominant processes driving the location where water flows during the waterfloods: gravity, capillarity, and the applied pressure gradient. For each of these samples, a different process is more dominant. In the Ksand (Figure 9), gravity is more important, with capillarity gaining importance particularly with hydrate present. This is expected, because of the relatively large grain sizes and high porosity. The presence of hydrate, a water-wetting solid phase, will reduce the pore dimensions increasing the strength of the capillary force. In the Fsand (Figure 11), capillary forces are more dominant. For the dry case, the effect of gravity is observed, but it is secondary to the capillarity. In the FsandSilt, the applied pressure gradient becomes dominant, particularly for the hydrate-bearing case. In the dry case, although water initially reached the slice at the bottom of the sample, the front is fairly vertical. The water flowing in the outer radii regions with lower hydrate saturation and where we assumed had higher permeability and porosity and lower capillary strength than the center, indicates the importance of the applied pressure gradient. This is more obvious in the hydrate-bearing case, in which the arrival time of the water flow at a particular location is proportional to the hydrate saturation (water arrives earlier at lower saturations).

Because of the large amount of data collected, we continue our analysis here on the Ksand experiments as an example but also describe the results from the other media. In Figure 13, we present the average water saturation over several cross sections for hydrate-bearing Ksand 28 and Ksand20 samples. In this and similar figures, the location (Loc) refers to the nominal location (in millimeters) from the starting scanning location. The maximum water saturation during a waterflood is limited by the saturations of hydrate and residual gas in the pore space. Water saturation recalculated considering hydrate as part of the solid phase $\left(\mathrm{S}_{\mathrm{w}}{ }^{*}\right)$ is presented on the right side of Figure 13. These plots indicate that in this hydrate-bearing sand, there is very little residual gas present after the water front passes a location. When presented in terms of $\mathrm{S}_{\mathrm{w}}{ }^{*}$, the curves are strongly similar to each other for the two conditions, but the slopes of the curves differ between Ksand 28 and Ksand 20. The slopes are steeper and tail more for the higher hydrate saturation (Ksand 28) because of the lower effective porosity. The stronger tailing indicates more heterogeneity as well, as verified by the hydrate saturation distribution (Figure 6).

Average water saturations at specific locations for waterfloods performed on dry samples (“dry” waterflood) for the Ksand20 and Ksand28 tests are presented in Figure 14. The shape of these curves is very similar to the waterfloods with hydrate present, yet note that the residual gas saturation for the dry waterfloods is much higher than with hydrate present. This trend was not always observed, however. For both FsandSilt and Fsand, $S_{w}{ }^{*}$ with hydrate exceeds $S_{w}$ for the dry (or low initial moisture content) waterfloods only for moderate hydrate saturations. For the higher and lower hydrate saturations, however, $\mathrm{S}_{\mathrm{w}}{ }^{*}$ did not exceed the dry sample waterflood $\mathrm{S}_{\mathrm{w}}$. This may be due to hydrate obstructing water flow when 
the saturation is high.

\subsection{Discussion \\ 4.1 Hydrate Formation}

Hydrate was expected to form more abundantly near the outer radii where heat transfer to the confining fluid was better. This was not the case: hydrate saturations were in general higher near the central axis of the sample (Figure 5). Three causes might be responsible for this: higher water saturation in the center because of water freezing and thawing, tighter grain packing in the center resulting in higher water saturation there, less time between ice melting and hydrate formation. The greater abundance of hydrate at the center is unlikely to be caused by increased water saturation in the center resulting from freezing and thawing. Comparisons of the pre-freeze and frozen sample CT data indicate that higher ice saturations were present near the outer radii, because the sample was cooled from the outside and freezing would occur first there. Water would then be imbibed towards the ice [Davis, 2001]. Comparisons of the pre-freeze and post-thaw images for Ksand 28 show slightly lower water saturation in the center and slightly greater water saturation at the sample bottom. Thus, the freezing and thawing did not result in higher water saturation in the center. The dry Ksand waterflood did not show enhanced imbibition into the center (Figure 9) as would be expected for tighter packing. Hydrate saturation was enhanced in the center of all Ksand samples (Figure 5) Therefore the better packing in the center is probably not the cause of enhanced hydrate formation there. During sample thawing, the last location where the frozen samples melted was in the center of the sample because of the radial heat transfer. The memory effect, in which partially organized water structures remaining from ice or dissociated hydrate promote hydrate nucleation [Schroeter et al., 1983; Sloan et al., 1998; Uchida et al., 2000b], may have been stronger in the center, since less time would have been available for this effect to decay. If this is the case, then nucleation might first occur in the center. Once that happens, hydrate growth induced water migration towards the hydrate.

\subsection{Permeability}

Each of the three media investigated behaved somewhat differently as the gas saturation changed as a result of phase changes (water, ice, hydrate). Because the density of water is $1 \mathrm{~g} / \mathrm{cm}^{3}$, and the densities of ice and hydrate are about $0.92 \mathrm{~g} / \mathrm{cm}^{3}$ [Sloan, 1990], the volume of the porespace occupied by the water-bearing phase changes by factors of 1 (water), 1.08 (ice) and 1.25 (hydrate). The reason for the difference between ice and hydrate is that in spite of their similar density, a mass of water will acquire additional mass from the incorporation of methane into the structure when hydrate is formed. Figure 8 shows measured gas relative permeabilities plotted against the gas saturation. With the exception of the water relative permeability at residual gas, which is not expected to follow this trend and the frozen Fsand28 case, the permeability declines with decreasing gas-phase saturation. The decline tends towards low values at a gas saturation of about $40 \%$. Lines have been added to Figure 8 to guide the eye in following the relative gas permeability of the moist sample, ice-bearing sample, and hydrate-bearing sample for the same sample. It is clear that each medium behaves differently. The gas relative permeability of the Ksand (black symbols and black linescoarse and most porous) declines most dramatically over the changes of phase. The FsandSilt samples changed more moderately, and the Fsand changed even less over the phase changes. The permeability of the frozen Fsand28 case does not fit with the behavior of the other samples. It is possible that moisture froze in the tubing causing this very low result. The CT scan of this condition did not reveal any clues.

To gain a perspective on the location of hydrate in the porespace of the samples (pore-filling or graincementing), we compare the water relative permeability of the water-satiated sample containing residual gas (but no hydrate), to the gas relative permeability of the hydrate-bearing systems. For the water relative permeability of the water-satiated sample containing residual gas, gas is the phase interfering with the flow. For the gas relative permeability of the hydrate-bearing samples, remaining water and hydrate are the phases interfering with water flow. In an unsaturated water-wet porous medium (such as silica sand with methane hydrate), water will be present in films coating the mineral grains and in pendular structures at the grain contacts. Upon waterflooding, water will be imbibed first into smaller pores and corners of pore bodies. Trapped gas will remain in the largest pore bodies and possibly in the center of pore throats. Flowing water must go around this gas, and thus it will be restricted to narrower (and thus more flowresistive) locations. The method of forming hydrate (excess gas) is thought to form hydrate preferentially at the grain contacts, and less so in the not in the pore bodies. This produces grain-cementing hydrate [Waite et al., 2004]. The water relative permeability for the water-satiated residual gas test (Ksand) was about 0.11 
for a gas (interfering phase) saturation of only about 28\%. For the wettest Ksand sample without hydrate $(\mathrm{Sw}=0.42)$, the gas relative permeability is about 0.55 (Figure 8$)$. This large difference in behavior is caused by the location of the interfering phase in the pore space. For Ksand 28, with an impeding phase saturation $\left(\mathrm{S}_{\mathrm{h}}+\mathrm{S}_{\mathrm{w}}=0.25+0.05=0.3\right.$ - similar in magnitude to that of the residual gas in Ksand 20) - the relative permeability was only $\sim 0.7$, far greater than for the residual gas case, in spite of the comparable interfering phase saturation. This comparison leads us to conclude that the hydrate formed in these tests did not primarily occupy the largest pore bodies (as gas would during a waterflood), but more likely tended to occupy locations similar to those occupied by the pendular water. This is consistent with SEM images of hydrate formed in this manner [Klapproth et al., 2006], and acoustic tests on similar samples [Waite et al., 2004; Winters et al., 2004].

The relative permeability of the hydrate-bearing sample for Ksand 20 was unexpectedly higher than the permeability of the moist sand. We explain this result by water migration during hydrate formation. Hydrate formation in the porous medium induces water flow towards it, similar to ice forming in freezing sediments [Davis, 2001], because the newly formed solids (hydrate) reduce the pore sizes and the smaller pores induce higher capillarity [Kneafsey et al., 2007; Gupta et al, 2006]. This happened in all these tests, but it was more severe relative to the initial saturation in Ksand 20. To examine the changes that occurred upon hydrate formation, we plotted a profile of the average hydrate saturations from the Ksand tests across the sample (from the rubber sleeve through the center to the rubber sleeve, Figure 15). As noted previously (e.g. Figure 5), the hydrate saturation in the center is higher than in the region closer to the rubber sleeve. Similarly, we plotted the profile of the change in non-gas saturation with hydrate present (called "Total Saturation” in Figure 15). In Ksand 42 and Ksand 28, the total saturation (hydrate + water) following hydrate formation generally exceeds the initial water saturation (Total Saturation Change is greater than zero). A substantial area within Ksand 20 has a lower total saturation after hydrate formation, as a result of water migration. Note that the saturation values closer to the rubber sleeve affect a larger area, due to their distance from the center and the cylindrical geometry.

\subsection{Interpretation of Results}

We compared our gas relative-permeability results to a number of models (Figure 16 and discussed further below). We constructed and evaluated three sinusoidally varying pore models each placing hydrate in a different location in the pore space. Kleinberg et al. [2003] summarized a number of permeability models having a single flowing phase, allowing for easy comparison and we included the (1) Coated Cylindrical Capillaries, (2) Hydrate Occupies Cylindrical Capillary Centers, (3) a Kozeny Grain Model, (4) Hydrate Occupies Pore Centers, and (5) the University of Tokyo Model with N=10 and 15.

\subsection{Sinusoidally Varying Radius Capillary Models}

We modeled a granular porous medium containing pore bodies and pore throats as an assembly of uniform parallel capillary tubes with sinusoidally varying cross section (Figure 17) where $r_{p}$ is used to denote the pore-body radius and $r_{t}$ is used to denote the pore-throat radius. Although capillaries having corners are thought to better model porous media [Blunt et al., 1995; Patzek and Kristensen, 2001; Ransohoff and Radke, 1988], we ignore the corners in this analysis for simplicity. We also assume that the flow capillaries are not interconnected, and that they are arranged compactly (hexagonal packing), with each pore body touching six nearest neighbors. We use the relative conductance of a single sinusoidal capillary as given by Bernabe and Olson [2000].

$$
\frac{g}{g_{o}}=\frac{2\left(1-\left(\alpha / r_{o}\right)^{2}\right)^{\frac{7}{2}}}{2+3\left(\alpha / r_{o}\right)^{2}}
$$

where $g_{o}=\frac{\pi r_{o}^{4}}{8 \mu}, \alpha$ is the amplitude of the cross-sectional variations, and $r_{o}$ is the average capillary radius.

In a partially water-saturated water-wetting porous medium, water is present as films on the mineral grains, and fills the pendular structures at the grain contacts. The pore body in our sinusoidally varying capillary corresponds to the pore body in a natural porous medium in which the water present is primarily in films. 
The pore throat in our sinusoidally varying capillaries corresponds to the pore throats in a porous medium, where water is more abundant in the pendular structures. If hydrate forms in both the pore bodies and pore throats (Case 1), the constriction in both will affect permeability. If hydrate forms preferentially in the pore bodies (Case 2), these large spaces will be relatively more constricted, gradually reducing permeability. If hydrate forms preferentially in the pore throats where the water is more abundant (Case 3), the pore throats will be more constricted, significantly affecting permeability at low saturation. Each affects permeability differently.

We define our original pore space such that the wavelength is twice the maximum pore-body radius, and the initial ratio of pore-throat radius to pore-body radius is $0.077 / 0.207$ [Berg, 1975]. When hydrate forms, we allow the original pore space to be modified in three ways: (1) the ratio of pore-throat radius $\left(\mathrm{r}_{\mathrm{t}}\right)$ and pore-body radius $\left(\mathrm{r}_{\mathrm{p}}\right)$ is constant (both pore body and pore throat fill but most filling is in the pore body corresponds to Case 1); (2) the pore-throat radius is kept constant and the interfering hydrate phase is contained in the pore body (Case 2); and (3) the maximum pore-body radius is held constant, and the interfering hydrate phase accumulates in the pore throat (Case 3). Graphical representations of these cases are shown in Figure 18, In Case 2, as the hydrate saturation increases, the pore body narrows until it reaches the throat radius, after which the resulting cylindrical tube constricts uniformly. In Case 3 , as the hydrate saturation increases, the pore throat narrows while the pore-body radius remains unchanged.

We also compare our data to the Millington-Quirk gas permeability model as modified by Moldrup [1998]. Our data, the computed gas relative permeability for the three sinusoidal varying pore models, the Millington-Quirk model, and other models are presented in Figure 16. Case 1 is identical to the MillingtonQuirk model that was derived for porous media. With both Cases 1 and 2, the gas relative permeability declines over the full range of impeding phase saturation. Although mathematically allowable, one would expect in reality that the relative gas permeability would become very low prior to reaching a gas saturation of zero, as seen in Moldrup [1998]. This is because capillary-held water will block flow paths at saturations lower than one.

The relative gas permeability for Case 2 is higher than for Case 1 because the pore throats are not affected in Case 2 until the pore body is filled. As expected, constricting the pore throats in Case 3 seals off the medium at very low saturation. Scaling the relative gas-permeability curve of Case 2 in Figure 16 to match the saturation where our permeability becomes very low provides a better, yet still imperfect, fit to the data. This scaling is justified, because no real system would become plugged up in a perfectly uniform manner (as in our mathematical model), and flow would undoubtedly be plugged well before reaching zero gas saturation. The rapid decrease in permeability with hydrate present near $50 \%$ saturation may indicate that pore throats become closed at this point. Since Case 3 does not fit the data well, it is likely that the hydrate in our case does not preferentially occupy only the pore throats.

Comparing the data to the models summarized by Kleinberg, the model providing the best fit to the data is the Hydrate Occupies Pore Center model (green line) over a large range of gas saturations. It was unexpected that the two best-fitting models are based on pore-filling-type models, because we assumed that the hydrate cements the grains together in the pore throat regions and is not primarily pore-filling. The Scaled Sinusoidal Pore Model Constant $\mathrm{r}_{\mathrm{t}}$ (black line) fits the data reasonably well. Scaling the model has the same effect as filling the pore space with porous hydrate such that it impedes flow prior to complete saturation.

To provide additional insight into the location of hydrate within the porespace, we compared the $\mathrm{S}_{\mathrm{w}}$ changes in the hydrate waterflood (Figure 13) to the dry waterflood (Figure 14). In the dry waterflood, the maximum $S_{w}$ was in the range of about 75 to $80 \%$. In the hydrate waterflood, when the hydrate is considered a part of the solid, $\mathrm{S}_{\mathrm{w}}{ }^{*}$ reaches more than $90 \%$. Considering our sinusoidal capillary-tube model, higher residual gas saturation will occur in a porous medium with a large ratio of pore-body radius to pore-throat radius. This implies that the hydrate decreases the ratio of the pore-body size to pore-throat size and that the hydrate tends to occupy the pore bodies.

\subsection{Conclusions}

The presence of hydrate in the pore space of a porous medium dramatically influences the flow of water 
and gas through that medium. In our tests, the permeability of our moist sands decreased when the water was frozen, and typically decreased further when hydrate was formed. At elevated hydrate saturations, hydrate significantly impacted permeability to the extent that we were unable to measure it with the equipment we were using. At lower initial water saturations, the formation of hydrate did not completely plug our samples, but the distribution of the hydrate in the sand altered the permeability. In one case (Ksand 20), hydrate formation increased the permeability of our sample compared to the moist condition in spite of the increased volume of interfering phase on hydrate formation, which occurred because the hydrate began forming nonuniformly in the porous medium.

Water flow through the hydrate-bearing samples was also significantly influenced by the presence of the hydrate. In some cases water flow was enhanced by increased capillary suction or deterred by the reduced permeability of the higher hydrate saturation regions. These observations are important for gas production from hydrate-bearing reservoirs, because regions containing elevated hydrate saturations will tend to imbibe water produced from nearby dissociating hydrate, reducing the gas permeability in these regions and possibly hindering gas production from them. This could happen volumetrically (water imbibed throughout the region) or in a shell around a region, with a shell being much less favorable, since less water would be needed to slow gas production.

More work is needed to understand how hydrate occupies the pore space in natural porous media and in laboratory samples, and how this affects transport parameters. Imaging work such as that done by Stern et al. [2004], Tohidi et al. [2001], Jin et al. [2005], and Tomutsa and Kneafsey (unpublished data); as well as studies that infer hydrate pore-occupancy behavior from other measurements such as this study, Kleinberg et al [2003], Winters et al. [2004] and Waite et al. [2004] have shed light on possible hydrate habits under specified conditions. However, more observations and corresponding measurements of permeability, relative permeability, and pore occupancy behavior (habit) are needed.

Comparing water permeability at residual gas to gas relative permeability in similarly occupied hydratebearing sands led us to infer that the hydrate did not in general behave like a residual gas in a watersaturated porous medium (i.e., primarily preferentially fill the largest pores). However, we have also inferred that hydrate does not only occupy pore throats. This leads us to conclude that the hydrate formed in our tests tends to form in the pore bodies (decreasing their size), but does not primarily fill the largest pores. Considering our simplistic gas relative permeability models, gas flow through our hydrate-bearing sands tends to be better modeled by hydrate that occupies smaller pore bodies and throats, and perhaps decreases the size of the larger pore bodies.

In this study, we used CT data, differential pressure, temperature, and mass balance for computing the relative permeability of gas and water in hydrate-bearing sediments, and used CT for the observation of water flow through hydrate-bearing and non-hydrate-bearing samples. The effect of hydrate saturation and distribution have been measured and observed for both gas and water flow. Numerical inversion of the CT and pressure data sets is now required to broaden and extend these measurements. Many more measurements are needed to examine the effect of grain size, grain wettability, and hydrate formation method.

\subsection{Acknowledgments}

The authors wish to acknowledge Lehua Pan and Dan Hawkes for their helpful comments in reviewing this manuscript. This work was supported by the Assistant Secretary for Fossil Energy, Office of Oil and Natural Gas, Office of Natural Gas and Petroleum Technology, through the National Energy Technology Laboratory, of the U.S. Department of Energy under Contract No. DE-AC02-05CH11231 and by the Korean Gas Hydrate Development Program of the Korea Institute of Geoscience and Mineral Resources (KIGAM). 
Table 1. Initial conditions for the permeability tests. Initially, the porespace is filled with water and gas. Following hydrate formation, hydrate, gas, and unconverted water are present.

\begin{tabular}{|c|c|c|c|c|c|c|c|c|}
\hline Sample & $\begin{array}{c}\text { Initial } \\
\text { Water } \\
\text { Saturation }\end{array}$ & $\begin{array}{c}\text { Porosity } \\
\text { (from CT) }\end{array}$ & $\begin{array}{c}\text { Hydrate } \\
\text { Saturation }\end{array}$ & $\begin{array}{c}\text { Gas } \\
\text { Saturation } \\
\text { with } \\
\text { Hydrate } \\
\text { Present }\end{array}$ & $\begin{array}{c}\text { Fraction of } \\
\text { Water } \\
\text { Converted } \\
\text { to Hydrate }\end{array}$ & $\begin{array}{c}\text { Sample } \\
\text { Diameter } \\
(\mathrm{cm})\end{array}$ & $\begin{array}{c}\text { Sample } \\
\text { Volume } \\
\left(\mathrm{cm}^{3}\right)\end{array}$ & $\begin{array}{c}\text { Sample } \\
\text { Length } \\
\text { (cm) }\end{array}$ \\
\hline Fsand28 & 0.28 & 0.31 & 0.35 & 0.65 & 1.00 & 5.25 & 789 & 36.5 \\
\hline Ksand20 & 0.20 & 0.38 & 0.21 & 0.75 & 0.82 & 5.89 & 969 & 35.6 \\
\hline Ksand28 & 0.28 & 0.38 & 0.31 & 0.63 & 0.82 & 5.78 & 945 & 36.0 \\
\hline Ksand42 & 0.42 & 0.38 & 0.36 & 0.50 & 0.67 & 5.71 & 917 & 35.9 \\
\hline FsandSilt23 & 0.23 & 0.31 & 0.26 & 0.71 & 0.89 & 5.28 & 291 & 13.3 \\
\hline
\end{tabular}

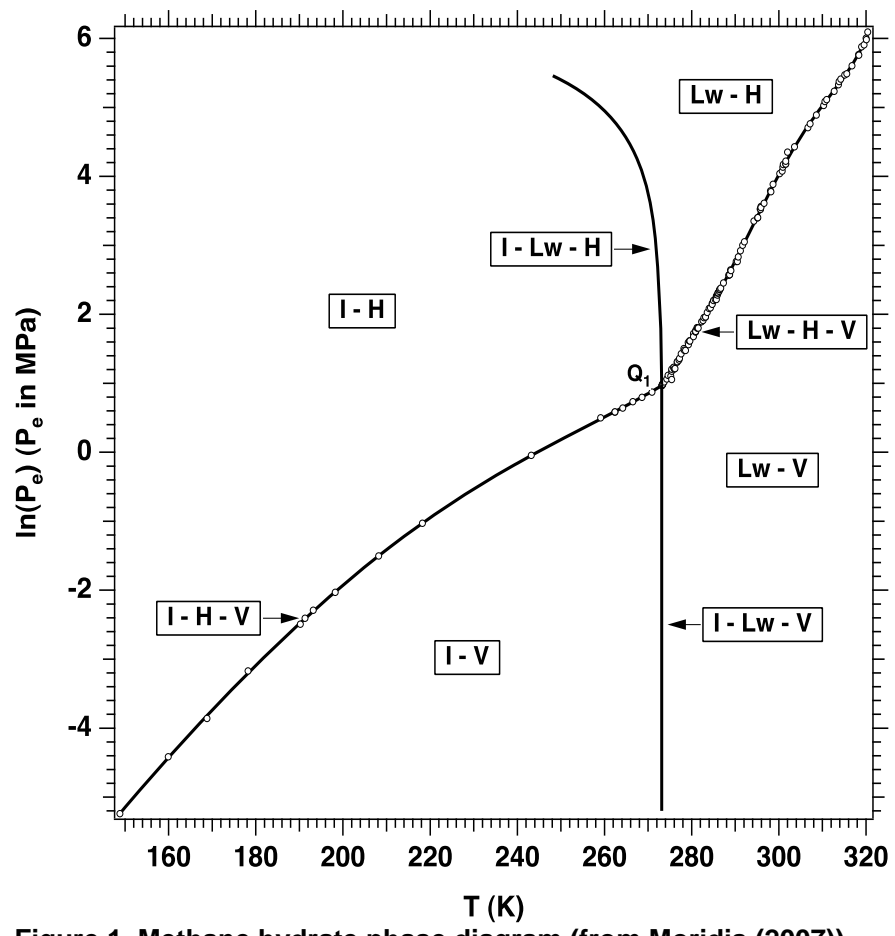

Figure 1. Methane hydrate phase diagram (from Moridis (2007)). 


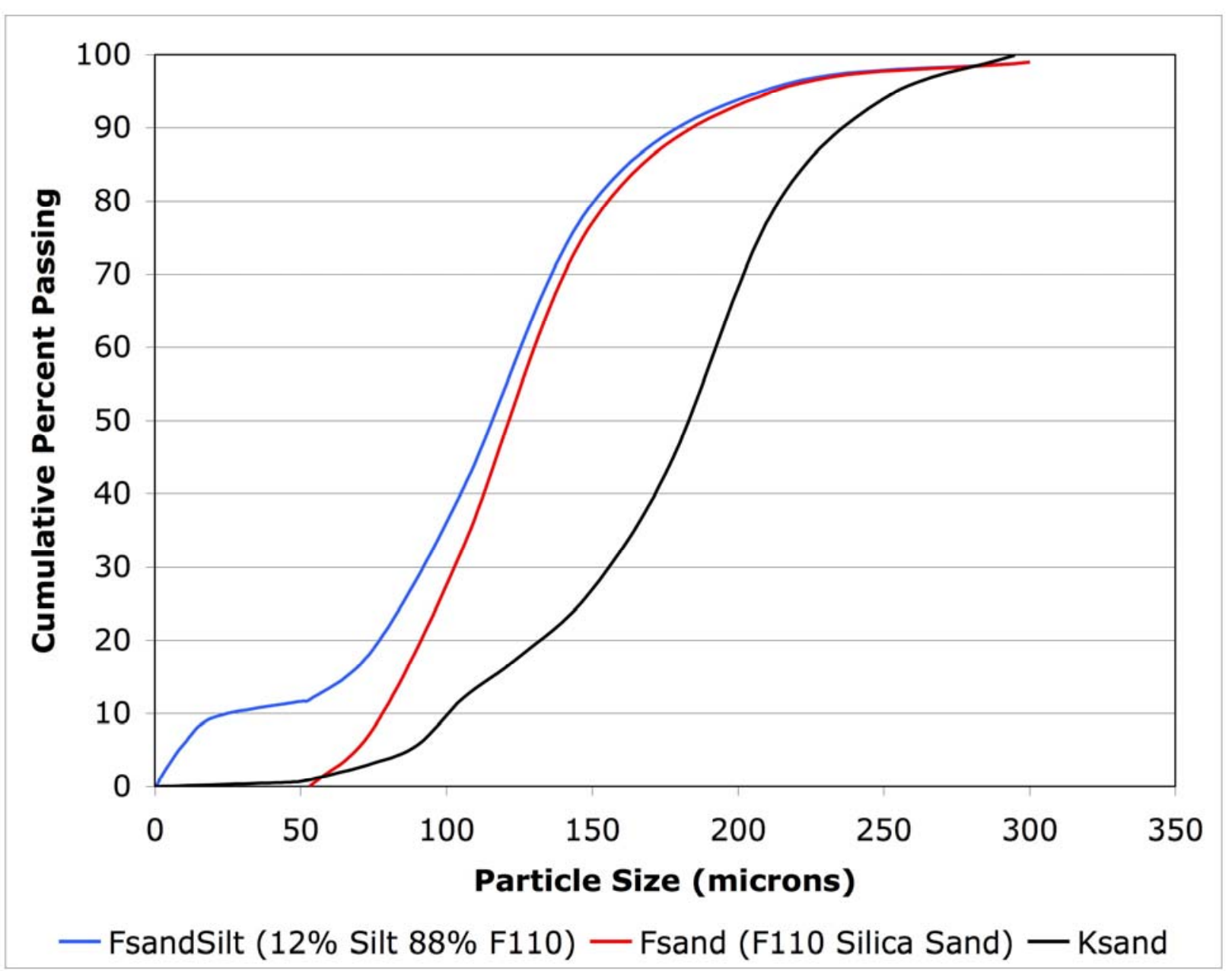

Figure 2. Sieve analyses 


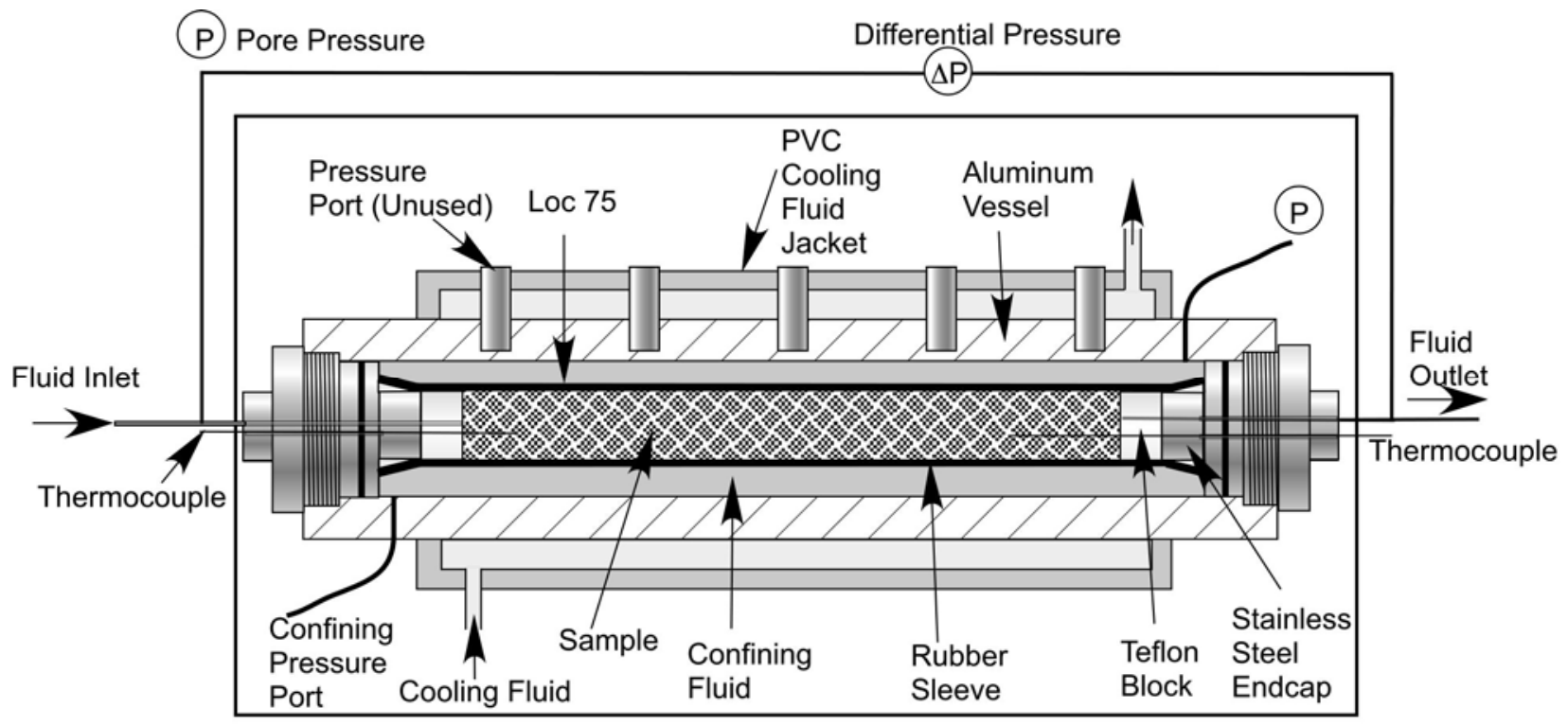

Insulated Box

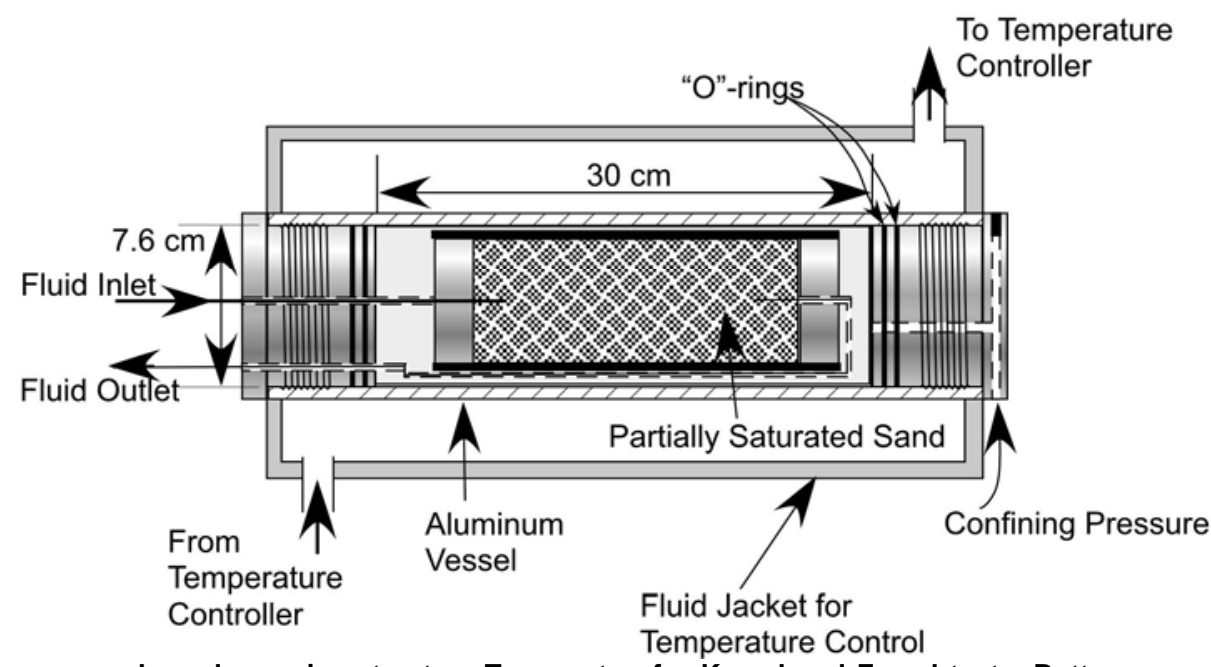

Figure 3. Pressure vessels and experiment setup. Top - setup for Ksand and Fsand tests, Bottom - vessel for FsandSilt tests (similar flow setup). 


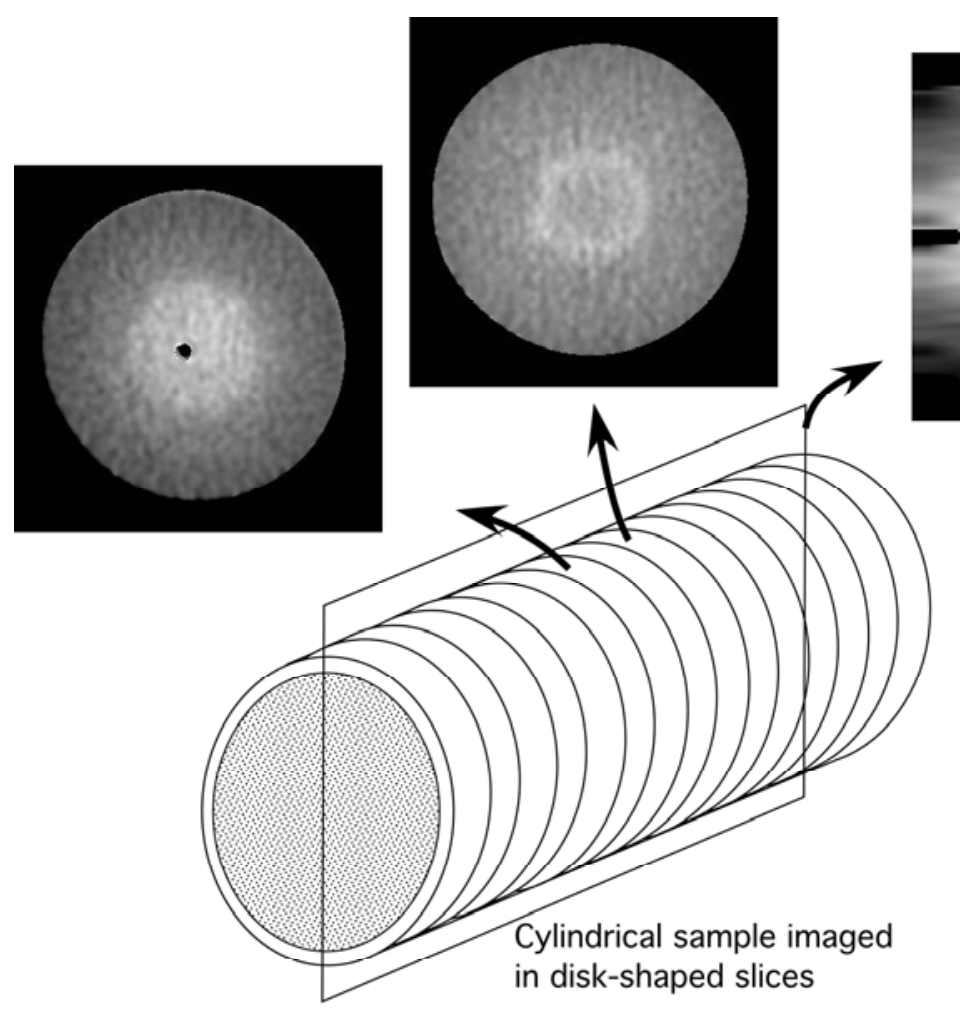

Axial spatial cross section Many slices at one condition

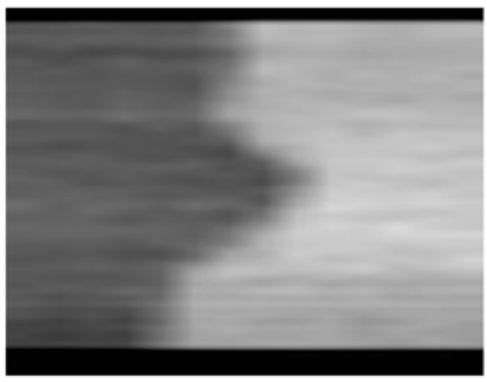

Temporal cross section

Same slice at different times

Figure 4. CT scanning of a cylindrical sample showing cross sections at various locations, and with time. Brighter regions indicate higher density. 
F110 Sand

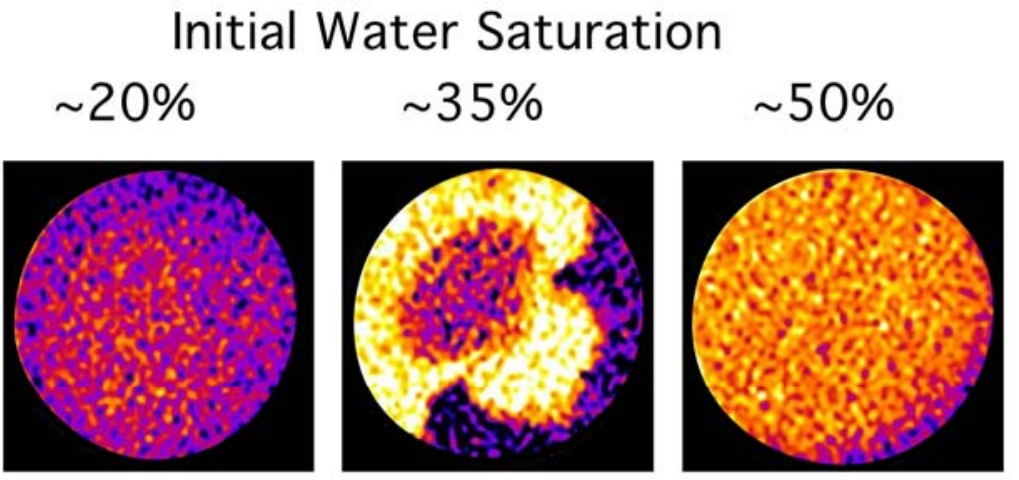

F110 Sand/Silt
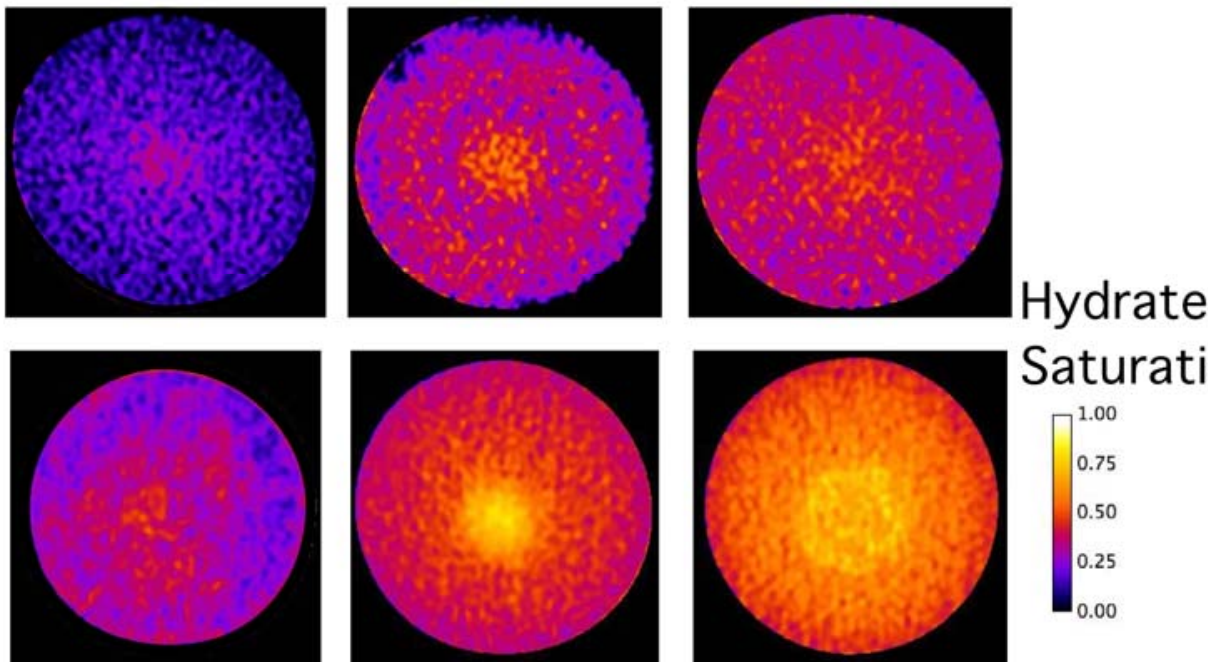

Saturation

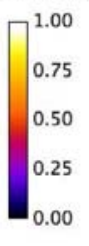

Figure 5. Characteristic hydrate saturations for the three initial conditions for each of the three porous media. Top (I to r) Fsand 28, Fsand 40, Fsand 60, Middle (I to r) Ksand 20, Ksand 28, Ksand 42, and Bottom (I to r) FsandSilt23, FsandSilt 39, FsandSilt 56. 


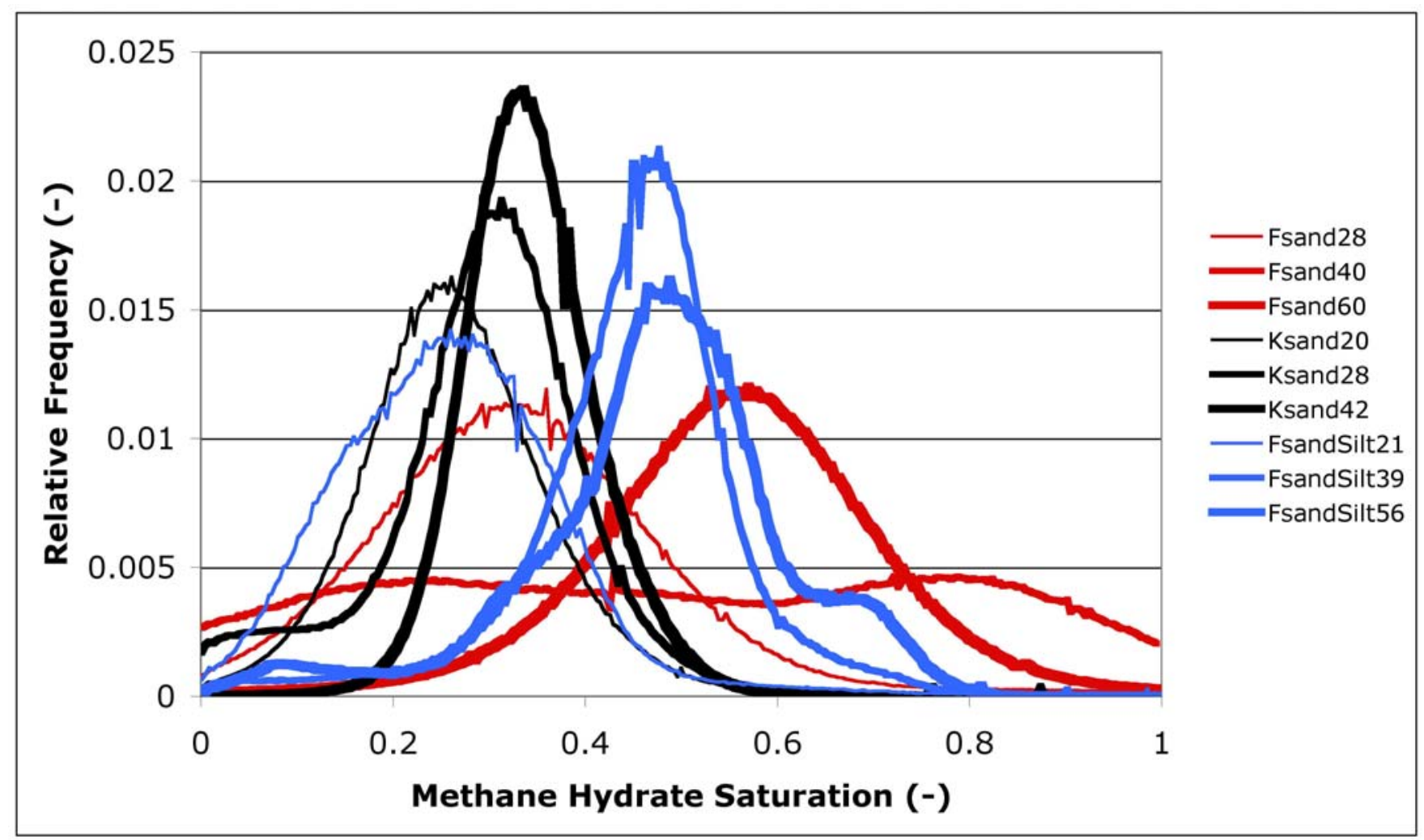

Figure 6. Hydrate saturation distribution in the 9 samples. 


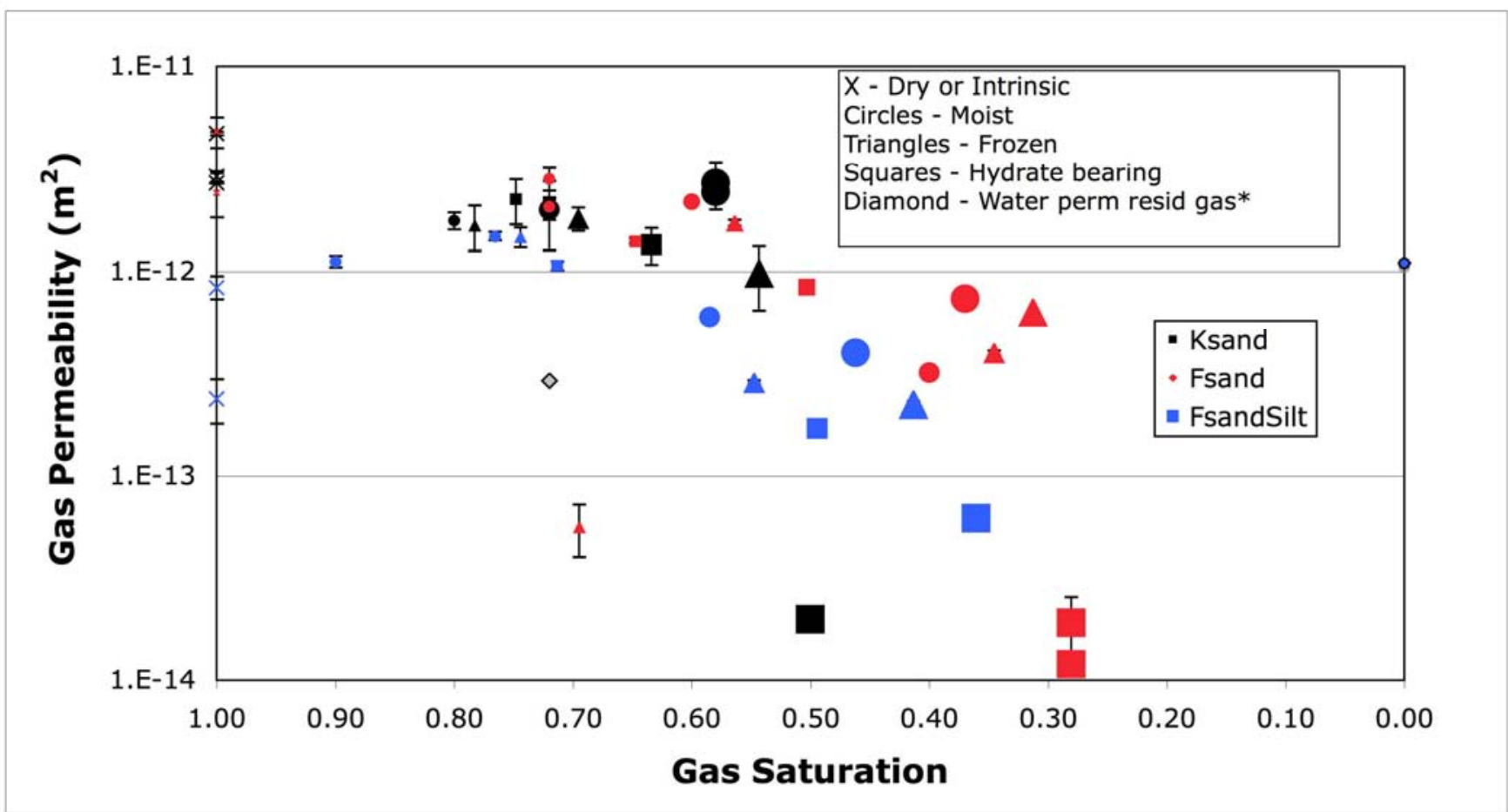

Figure 7. Sample permeabilities under various conditions. Smallest symbols indicate lowest initial water saturation, largest symbols indicate largest initial water saturation. For the Fsand, results from an additional moist and frozen sample are also plotted here. *Water permeability at residual gas saturation for Ksand28 is plotted (grey diamond) with the flowing phase (water) saturation plotted as the gas saturation.

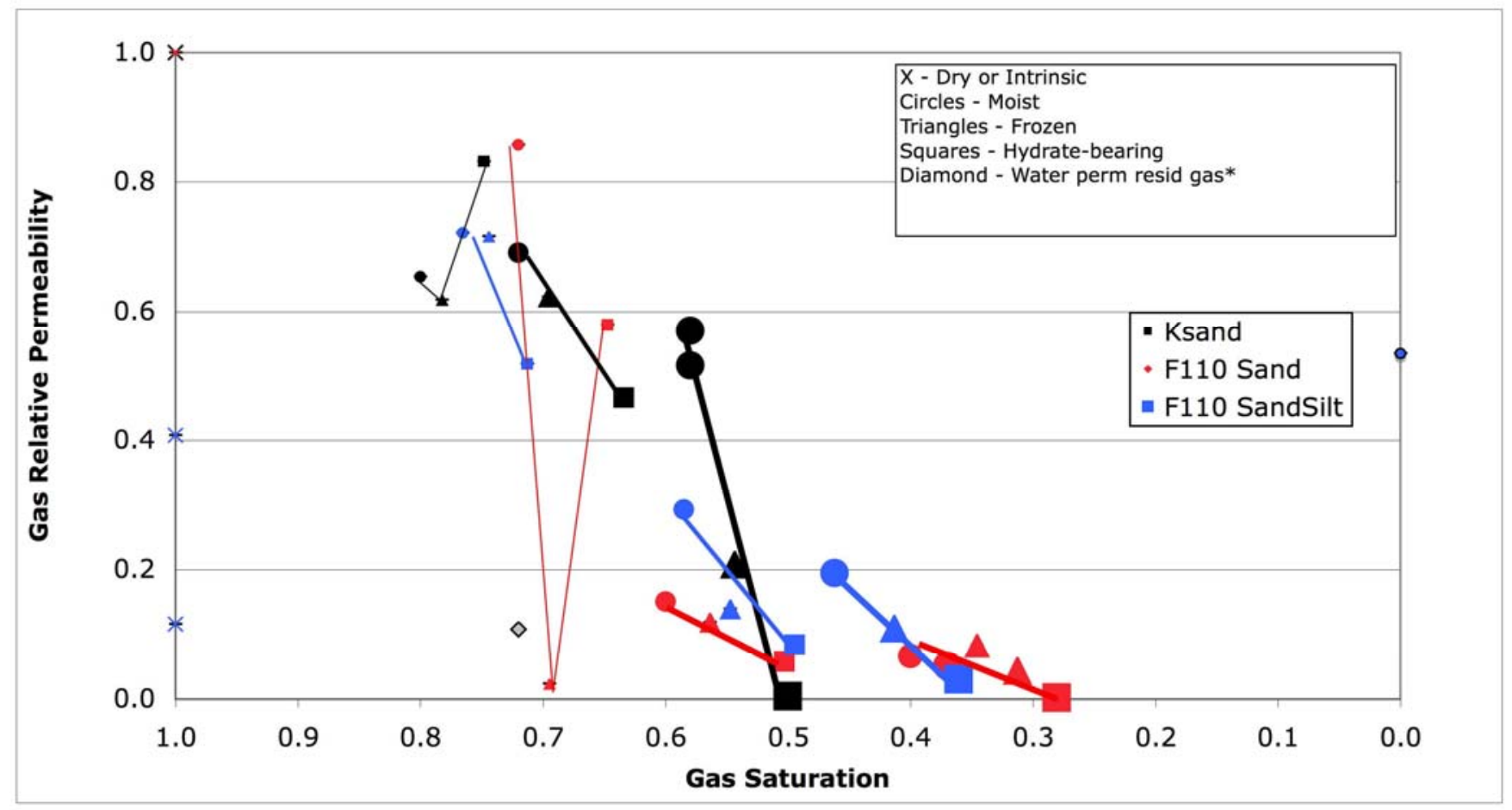

Figure 8. Gas relative permeability for stated conditions. Smallest symbols indicate lowest initial water saturation, largest symbols indicate largest initial water saturation. For the Fsand, results from an additional moist and frozen sample are also plotted here. Water permeability at residual gas saturation for Ksand28 is plotted (grey diamond) with the flowing phase (water) saturation plotted as the gas saturation. Lines were added to guide the eye through the series of conditions from moist to frozen to hydrate bearing. 

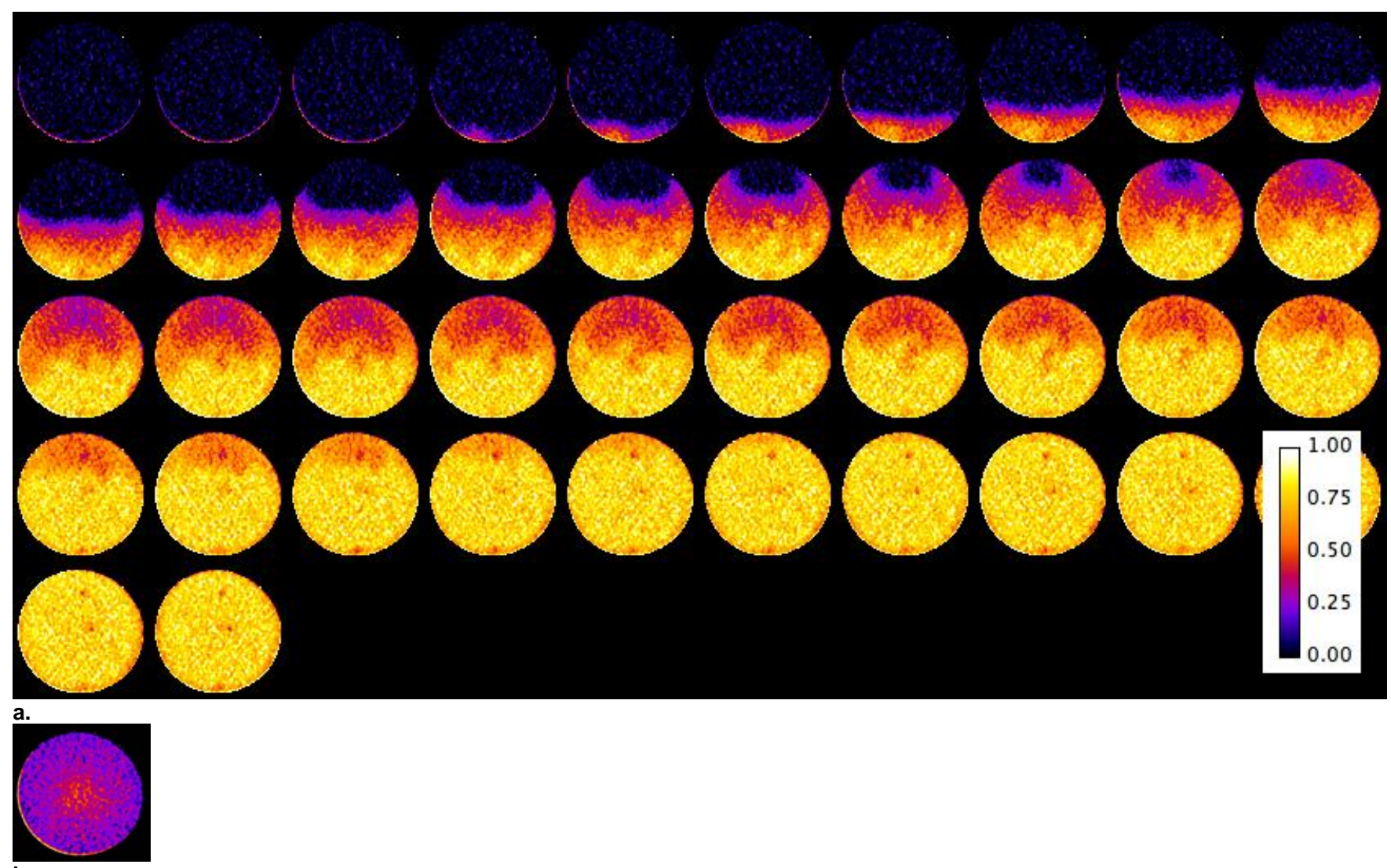

b. 


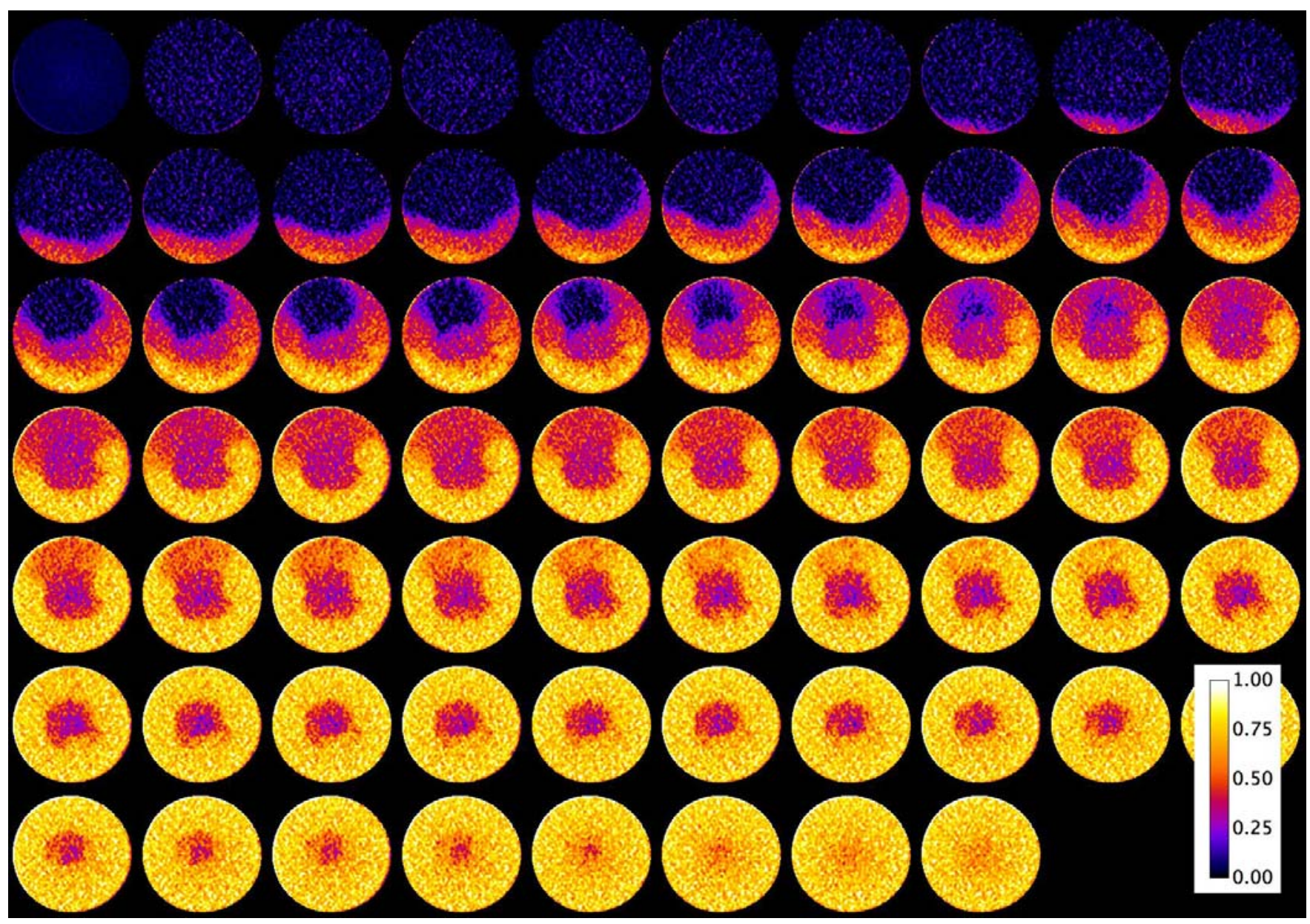

c.

Figure 9. a. Water saturations over time at Location 75 during the waterflood of dry Ksand, b. hydrate saturation at Location $\mathbf{7 5}$ for the waterflood under hydrate-bearing conditions for Ksand 20 (same color bar from a or $\mathrm{c}$ applies), and c. water saturations at Location 75 for the hydrate-bearing sample over the duration of the waterflood (approximately 3 hours). These circular slices were captured sequentially at the same location over the waterflood duration. Location 75 is located about $65 \mathrm{~mm}$ from the end of the Teflon end piece. (see Figure 3 , top). 


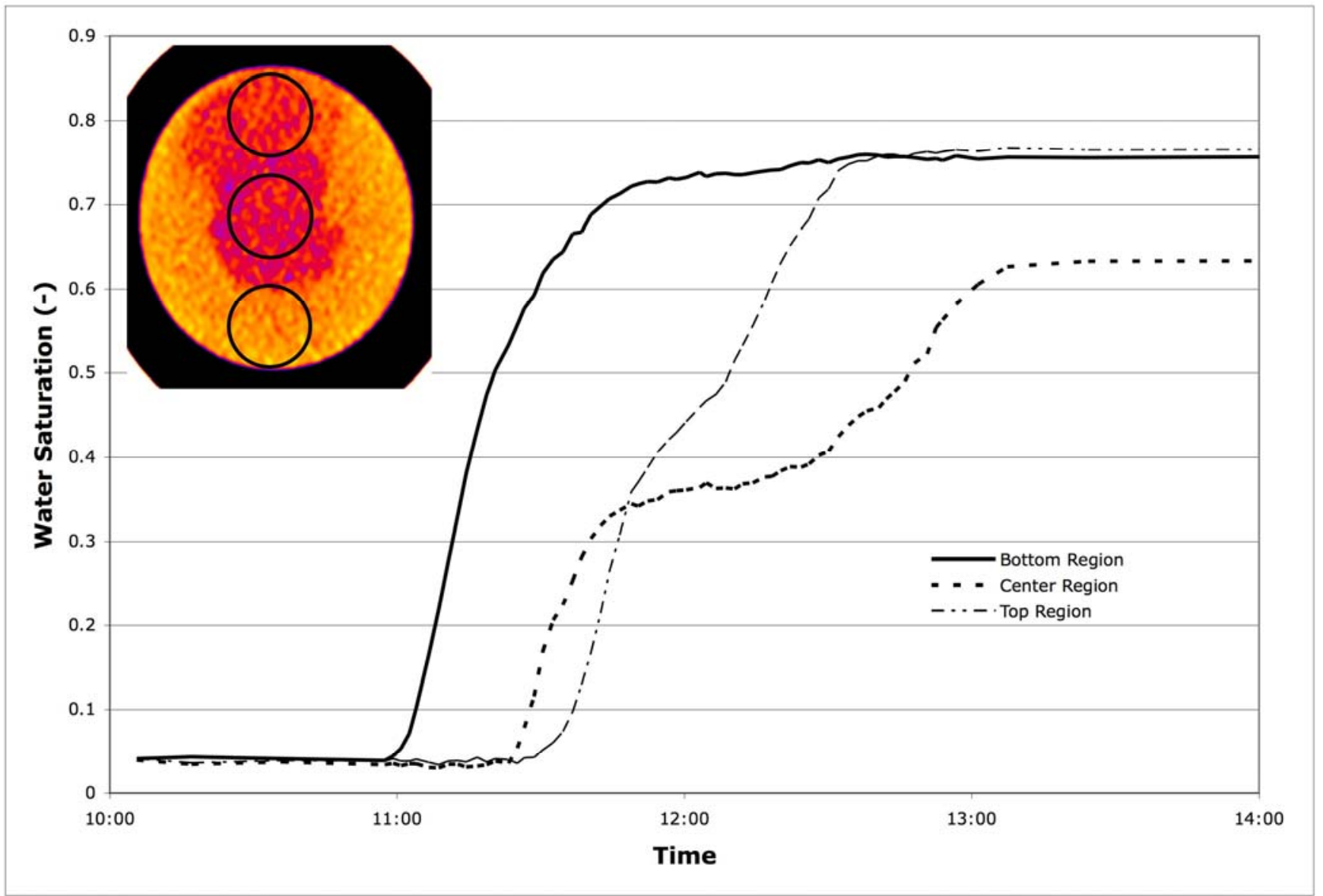

Figure 10. Location-specific water saturation changes at Location 75 in Ksand 20. The approximate average hydrate saturations in the top, middle, and bottom regions are $0.25,0.39$, and 0.27 . 


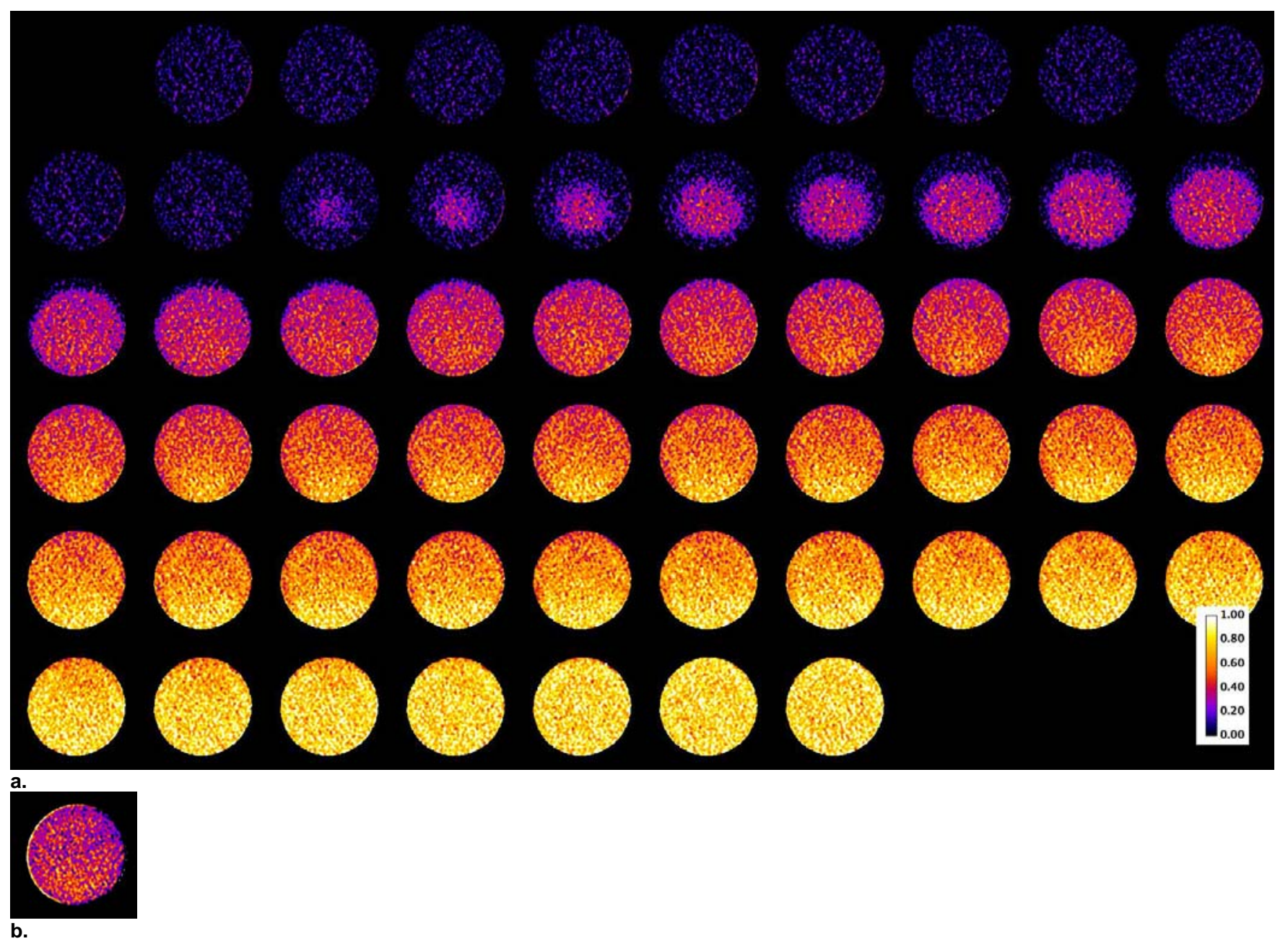




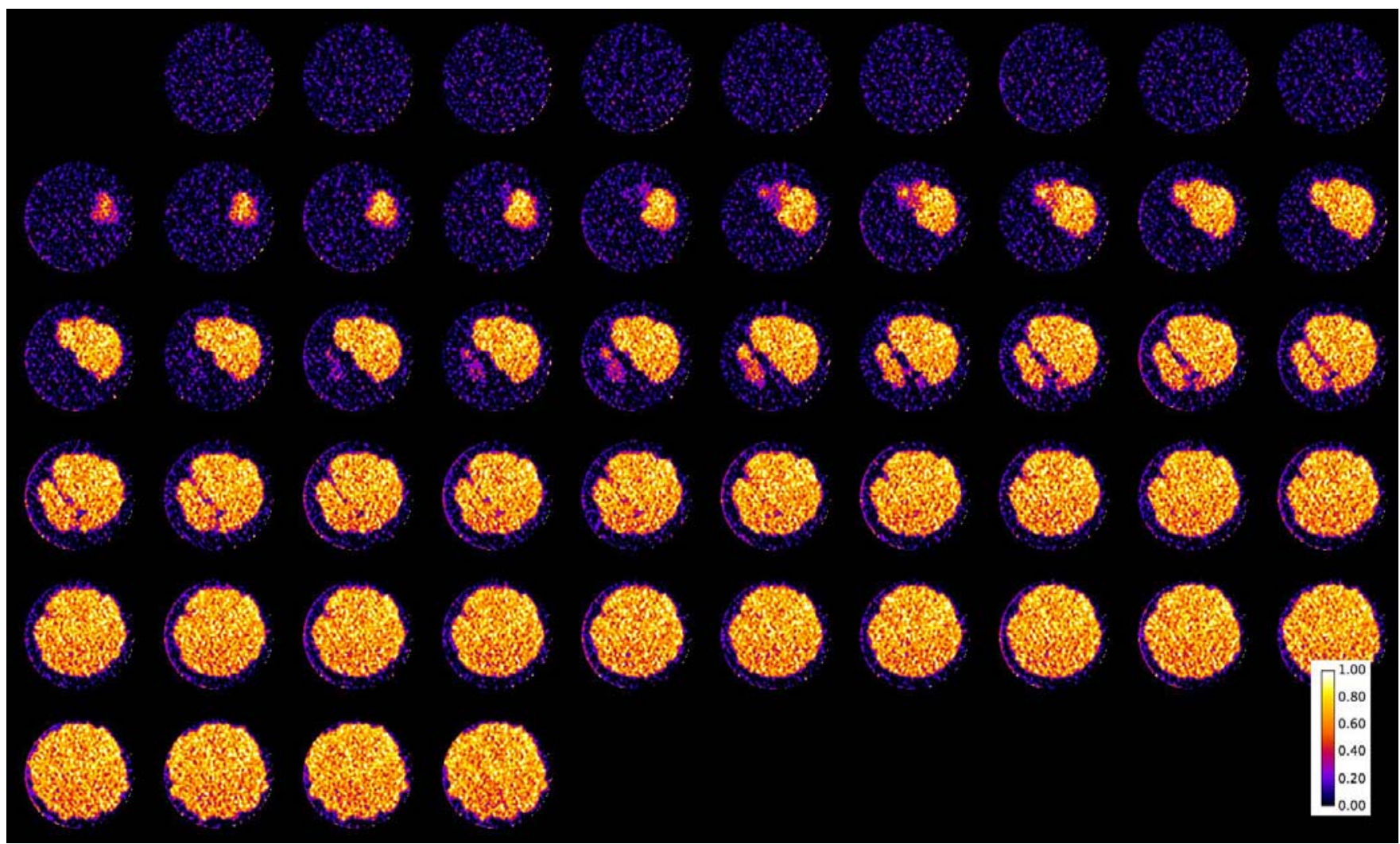

c.

Figure 11. Water saturation during waterflood in a. dry Fsand at Location 125, b. hydrate saturation at Location 125 (same color bar from a or c applies) and c. water saturation during the waterflood at Location 125 in hydratebearing Fsand28 (duration $\sim 3$ hours). 

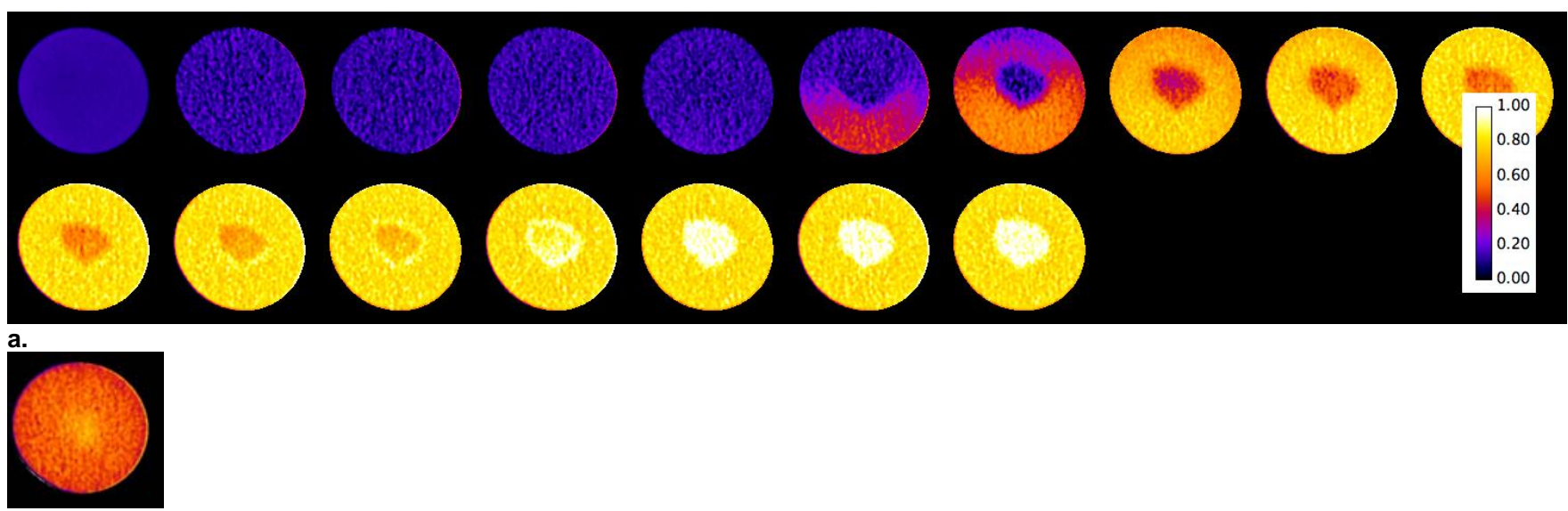

b.

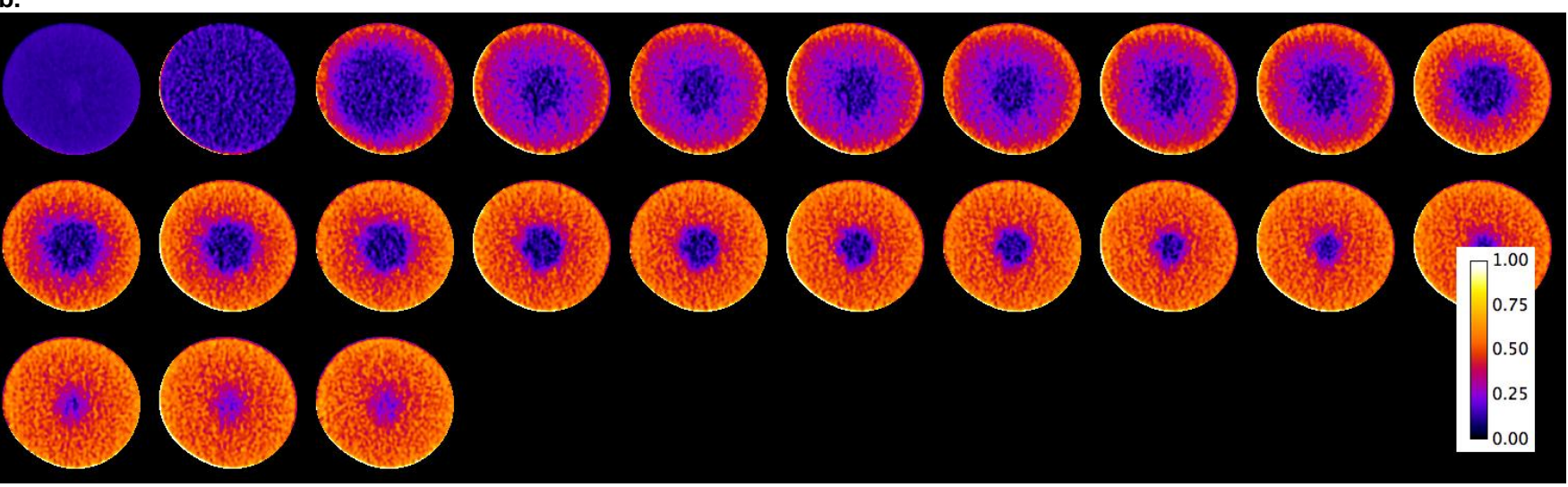

c.

Figure 12. a. Water saturations during the waterfloods of FsandSilt with $11 \%$ initial water saturation at Location 295 , b. hydrate saturation at Location 340 in FsandSilt39 (same color bar from a or c applies), and c. water saturations during waterflood of FsandSilt 39 with hydrate at Location 340 (duration $\sim 1.7$ hours). 


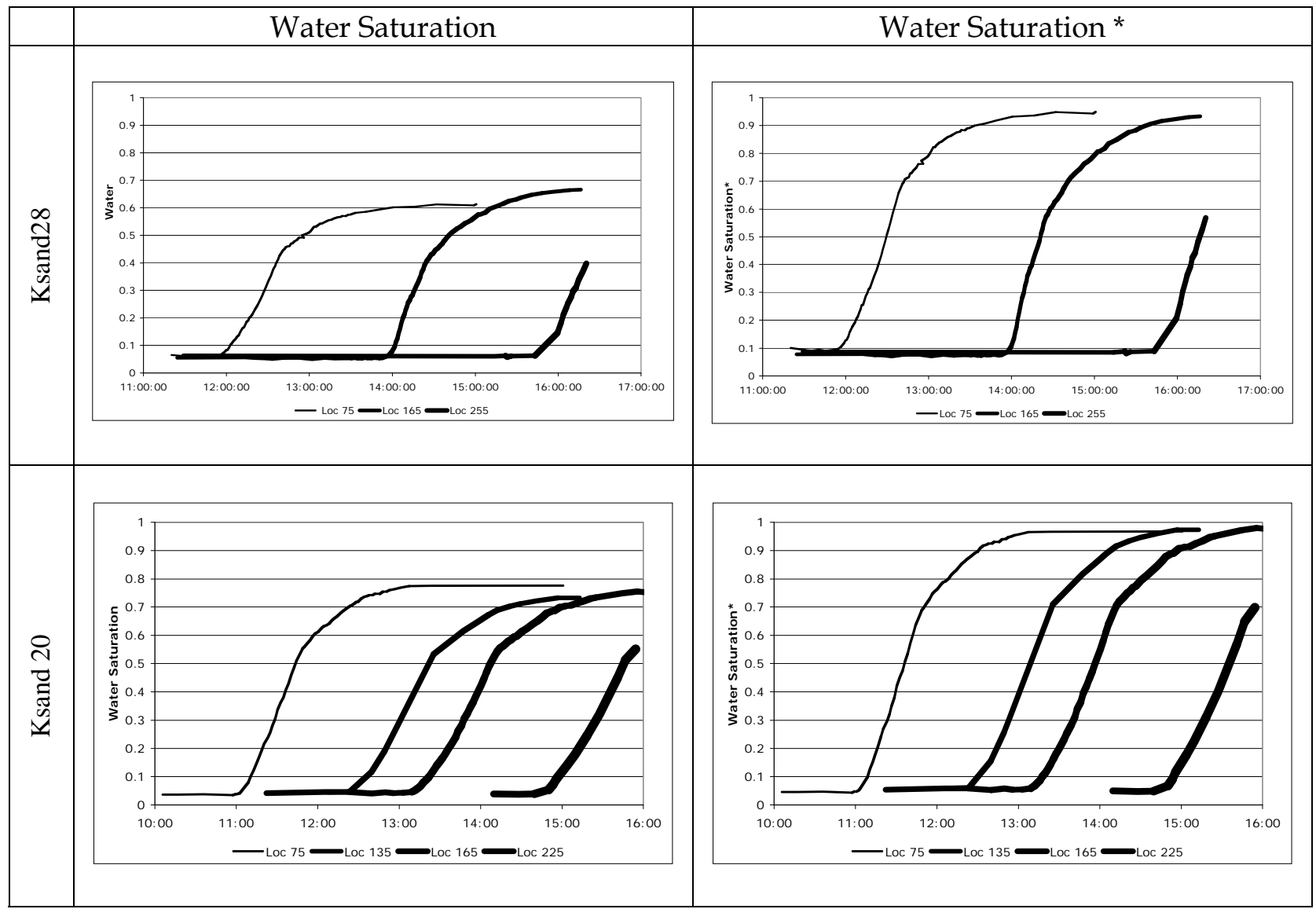

Figure 13. Average water saturations at specific locations (left column) and Water Saturation* (considers hydrate part of the solid medium) during the waterflood for Ksand28 and Ksand 20 The horizontal axis is time, and the locations scanned (Loc) are in $\mathrm{mm}$ from the initial scanning location. Loc 75 is $65 \mathrm{~mm}$ from the inlet, Loc 135 is $125 \mathrm{~mm}$ from the inlet,...

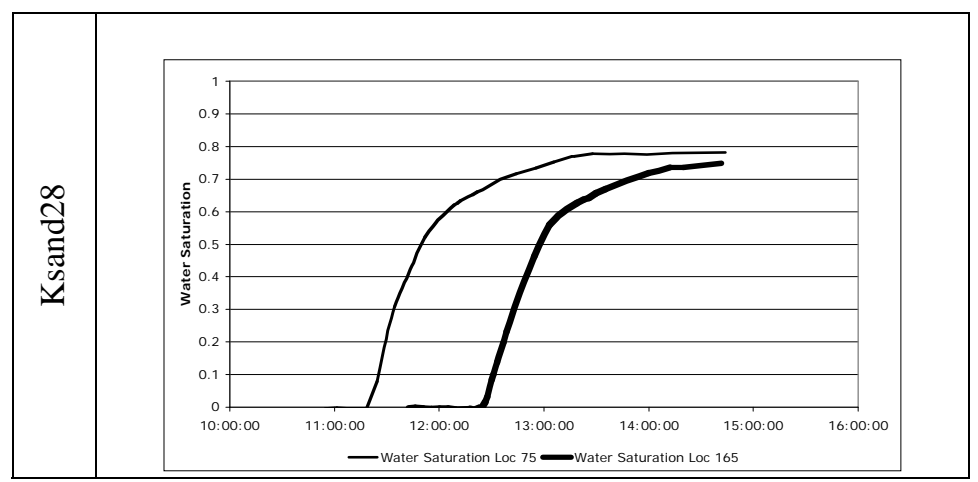




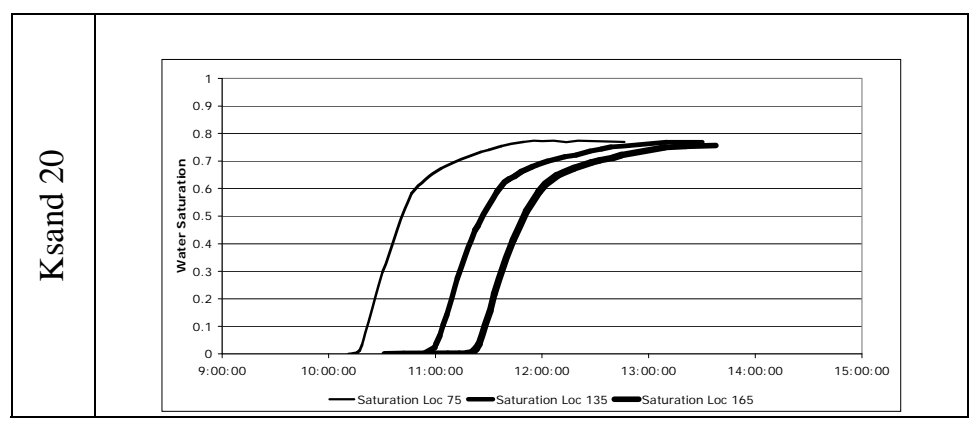

Figure 14. Average water saturations at locations during the "dry" waterfloods for Ksand28 and Ksand 20. The horizontal axis is time, and the locations scanned (Loc) are in $\mathrm{mm}$ from the initial scanning location. 


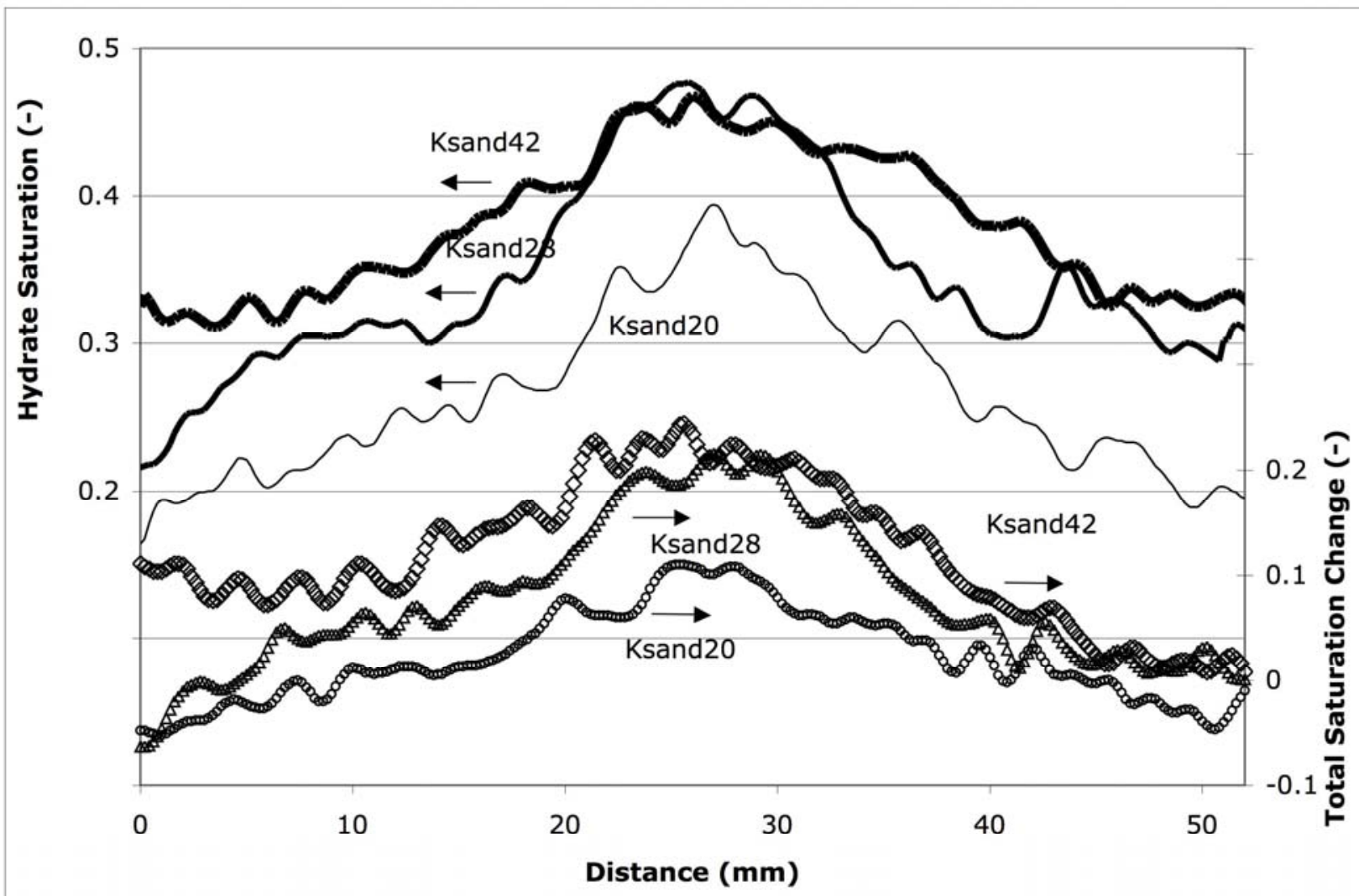

Figure 15. Average hydrate saturation profiles (lines) from rubber sleeve through the center (high saturation) to the rubber sleeve) and change in total saturation upon hydrate formation (symbols). 


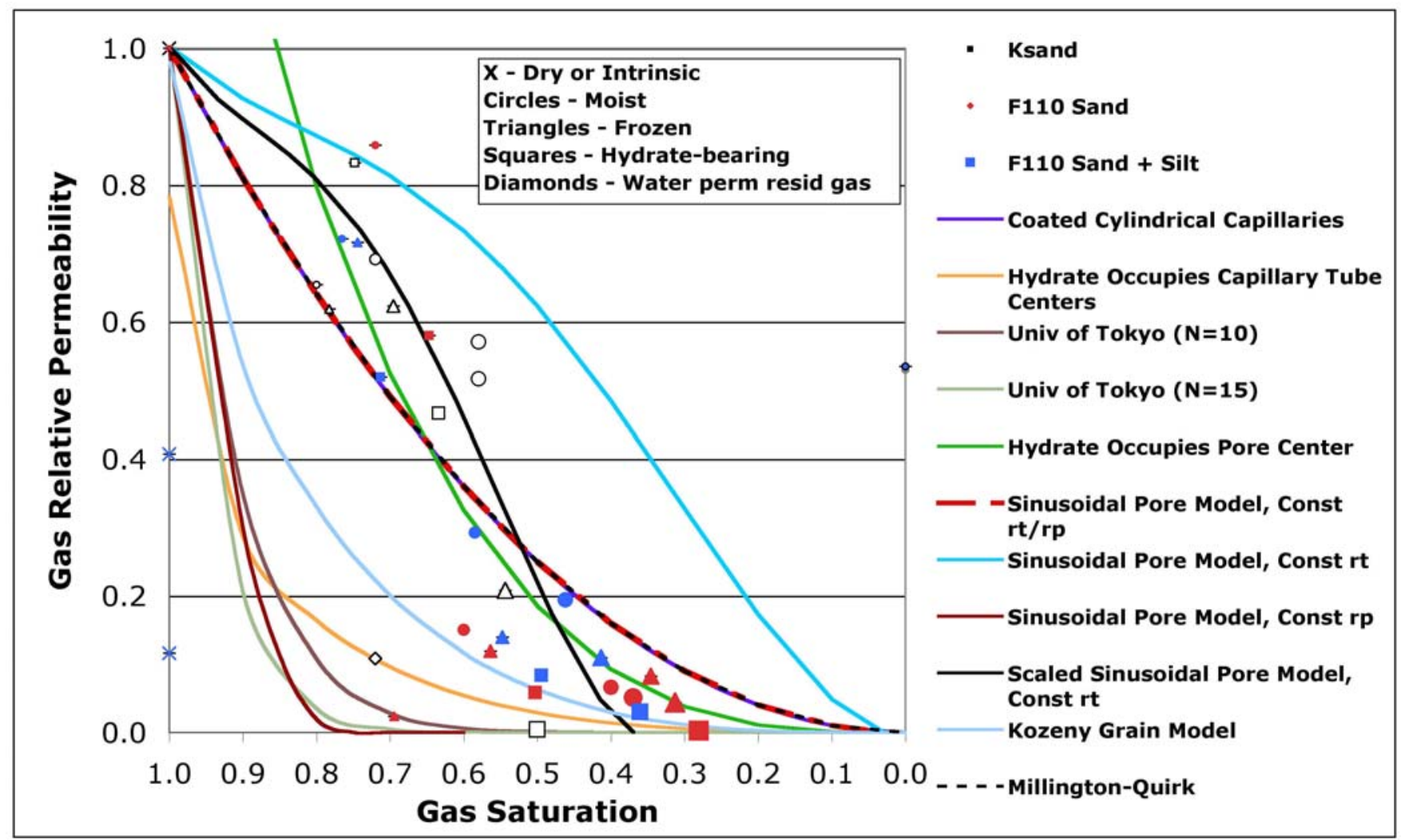

Figure 16. Gas relative permeability for stated conditions and relative permeability models. Smallest symbols indicate lowest initial water saturation, largest symbols indicate largest initial water saturation. 
a)

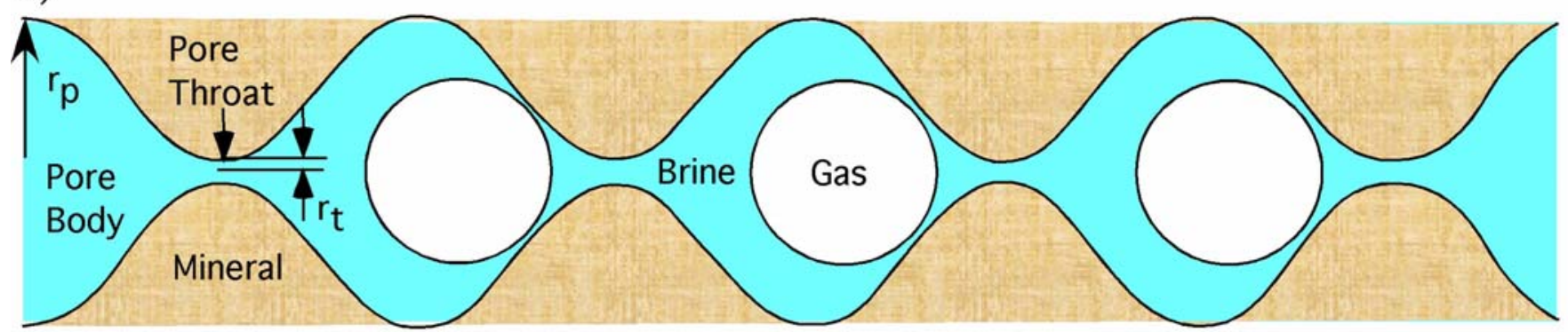

b)

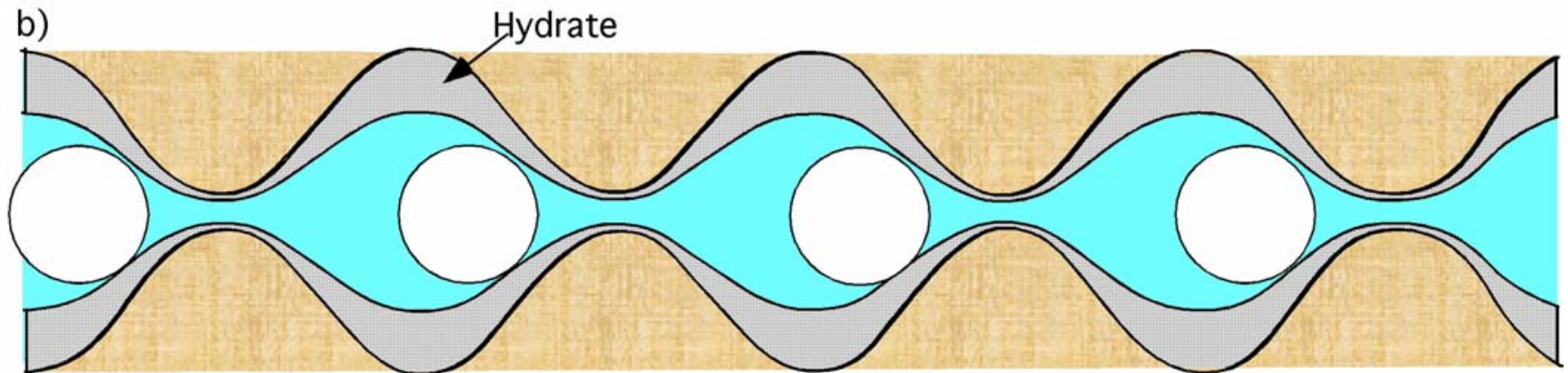

Figure 17. Sinusoidal porespace model and residual gas entrapment 


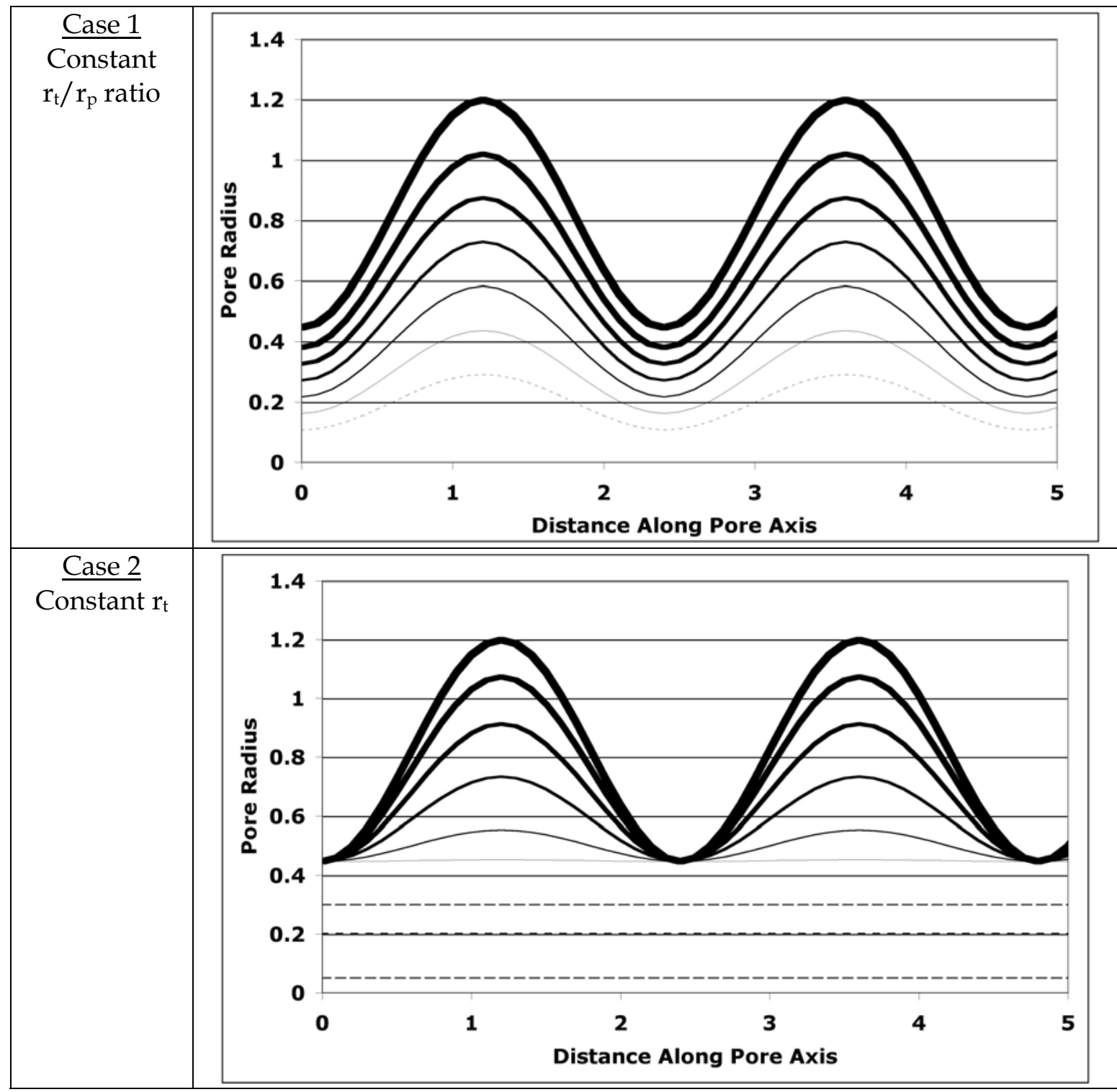




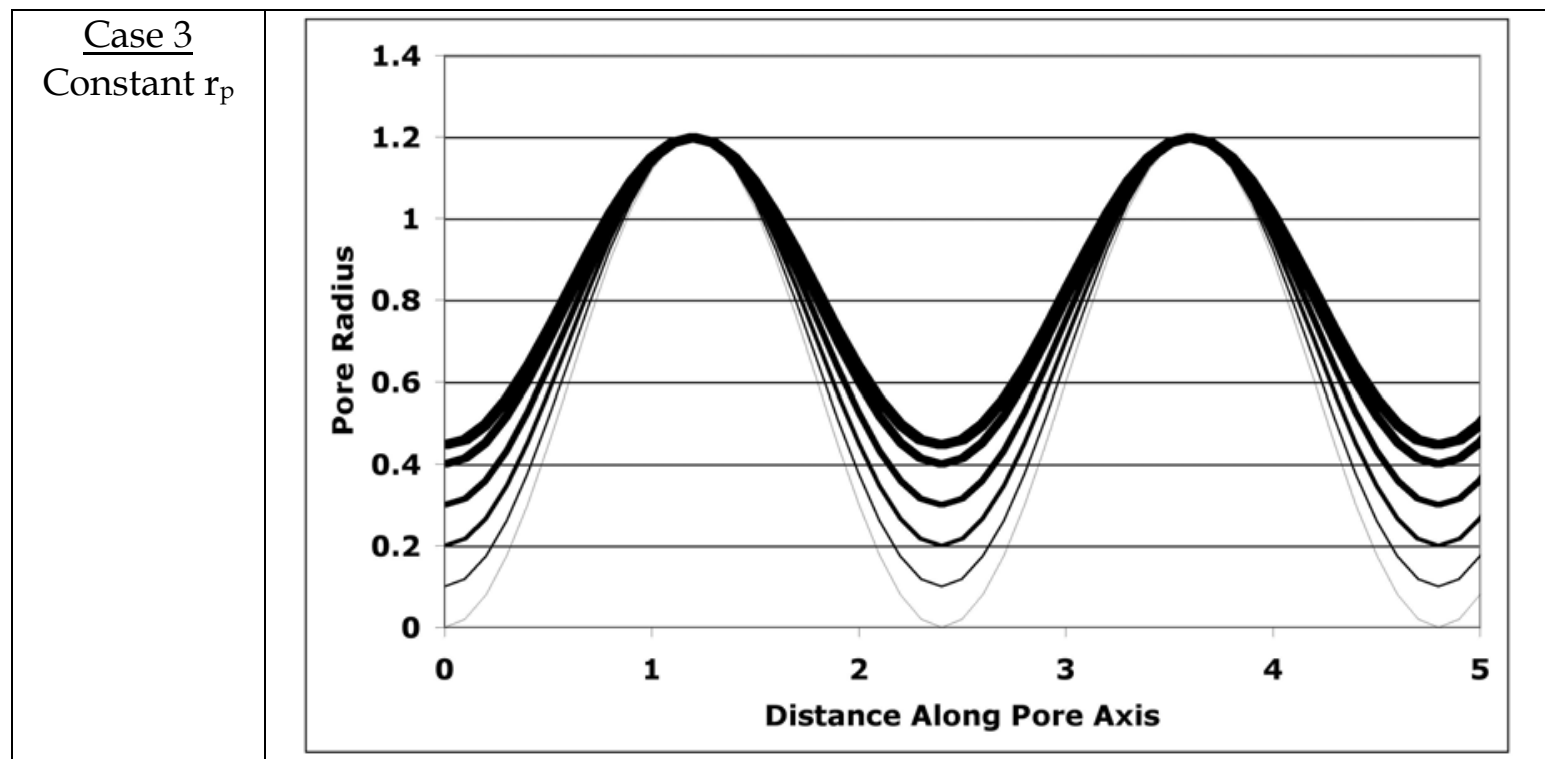

Figure 18. Pore geometry changes investigated in sinusoidally varying cross section models. 


\section{References}

Berg, R. R. (1975), Capillary pressure in stratigraphic traps, American Association of Petroleum Geologists Bulletin, 59, 939-956.

Bernabe, Y., and J. F. Olson (2000), The hydraulic conductance of a capillary with a sinusoidally varying cross-section, Geophysical Research Letters, 27(2), 245-248.

Blunt, M., et al. (1995), Three-phase flow and gravity drainage in porous media, Transport in Porous Media, 20(1/2), 77-103.

Davis, T. N. (2001), Permafrost: a guide to frozen ground in transition, University of Alaska Press, Fairbanks, Alaska.

Gupta, A., et al. (2006), Composite thermal conductivity in a large heterogeneous porous methane hydrate sample, J Phys Chem B., 110(33), 16384-16392.

Handa, Y. P., and D. Stupin (1992), Thermodynamic Properties and Dissociation Characteristics of Methane and Propane Hydrates in 70A Radius Silica Gel Pores, J. Physical Chemistry, 96, 8599.

Jaiswal, N. J. (2004), Measurement of Gas-Water Relative Permeabilities in Hydrate Systems, Masters of Science thesis, University of Alaska, Fairbanks, AK.

Jin, S., et al. (2005), Structure analysis of methane hydrate sediments by microfocus X-ray CT, in Fifth International Conference on Gas Hydrates, edited, Trondheim, Norway.

Kerkar, P., et al. (2009), Direct observations of three dimensional growth of hydrates hosted in porous media, Applied Physics Letters, 95(2), 024102-024103.

Klapproth, A., et al. (2006), Microstructure of gas hydrates in porous media, in 11th International Conference on the Physics and Chemistry of Ice, edited, Royal Society of Chemistry, Bremerhaven.

Kleinberg, R. L., et al. (2003), Deep sea NMR: Methane hydrate growth habit in porous media and its relationship to hydraulic permeability, deposit accumulation, and submarine slope stability, JGR, 108(B10), 2508.

Kneafsey, T. J., et al. (2007), Methane Hydrate Formation and Dissociation in a Core-Scale Partially Saturated Sand Sample, JPSE, 56, 108-126.

Kumar, A., et al. (2010), Experimental determination of permeability in the presence of hydrates and its effect on the dissociation characteristics of gas hydrates in porous media, JPSE, 70, 114-122.

Lemmon, E. W., et al. (2005), Thermophysical Properties of Fluid Systems - NIST Chemistry WebBook, NIST Standard Reference Database Number 69, in http://webbook.nist.gov, edited, National Institute of Standards and Technology.

Minagawa, H., et al. (2005), Water permeability measurements of gas hydrate-bearing sediments, in Fifth International Conference on Gas Hydrates, edited, p. 1058, Trondheim, Norway.

Moldrup, P., et al. (1998), Gas permeability in undisturbed soils: measurements and predictive models, Soil Science, 163(3), 180-189.

Moridis, G., et al. (2007), Depressurization-Induced Gas Production From Class 1 Hydrate Deposits, SPE Reservoir Evaluation \& Engineering, 10(5), 458-481.

Patzek, T. W., and J. G. Kristensen (2001), Shape factor correlations of hydraulic conductance in noncircular capillaries, JCIS, 236(2), 305-317.

Ransohoff, T. C., and C. J. Radke (1988), Laminar Flow of a Wetting Liquid Along the Corners of Predominantly Gas-Occupied Noncircular Pore, JCIS, 121(2), 392-401.

Schroeter, J. P., et al. (1983), Hydrate Decomposition Conditions in the System H2S-Methane-Propane, Ind. Eng. Chem. Fundam., 22, 361-364.

Sloan, E. D. (1990), Clathrate Hydrates of Natural Gases, Marcel-Dekker, Inc, New York, 664.

Sloan, E. D., et al. (1998), Quantifying Hydrate Formation and Kinetic Inhibition, Ind. Eng. Chem. Res., 37, 3124-3132.

Spangenberg, E., and J. Kulenkampff (2005), Physical properties of gas hydrate-bearing sediments, in Fifth International Conference on Gas Hydrates, edited, p. 2028, Trondheim, Norway.

Stern, L. A., et al. (1996), Peculiarities of methane clathrate hydrate formation and solid-state deformation, including possible superheating of water ice, Science, 273(5283), 1843-1848.

Stern, L. A., et al. (2004), Scanning electron microscopy investigations of laboratory-grown gas clathrate hydrates formed from melting ice, and comparison to natural hydrates, American Mineralogist, 89(89), 1162-1175.

Tohidi, B., et al. (2001), Visual observation of gas-hydrate formation and dissociation in synthetic porous media by means of glass micromodels, Geology, 29(9), 867-870. 
Uchida, T., et al. (2000a), Occurrences of natural gas hydrates beneath the permafrost zone in Mackenzie Delta visual and X-ray CT imagery, Annals of the New York Academy of Sciences, 912(Gas Hydrates), 1021-1033.

Uchida, T., et al. (2000b), Observations of CO2-hydrate decomposition and reformation processes, Journal of Crystal Growth, 217, 189-200.

Waite, W. F., et al. (2002), Thermal conductivity measurements in porous mixtures of methane hydrate and quartz sand, Geophysical Research Letters, 29(24), 2229, doi:2210.1029/2002GL015988.

Waite, W. F., et al. (2004), Methane hydrate formation in partially water-saturated Ottawa sand, American Mineralogist, 89, 1202-1207.

Waite, W. F., et al. (2008), Physical property changes in hydrate-bearing sediment due to depressurization and subsequent repressurization, JGR, 113(B07102).

Winters, W. J., et al. (2004), Physical properties and rock physics models of sediment containing natural and laboratory-formed methane gas hydrate, American Mineralogist, 89, 1221-1227. 


\section{DISCLAIMER}

This document was prepared as an account of work sponsored by the United States Government. While this document is believed to contain correct information, neither the United States Government nor any agency thereof, nor The Regents of the University of California, nor any of their employees, makes any warranty, express or implied, or assumes any legal responsibility for the accuracy, completeness, or usefulness of any information, apparatus, product, or process disclosed, or represents that its use would not infringe privately owned rights. Reference herein to any specific commercial product, process, or service by its trade name, trademark, manufacturer, or otherwise, does not necessarily constitute or imply its endorsement, recommendation, or favoring by the United States Government or any agency thereof, or The Regents of the University of California. The views and opinions of authors expressed herein do not necessarily state or reflect those of the United States Government or any agency thereof or The Regents of the University of California.

Ernest Orlando Lawrence Berkeley National Laboratory is an equal opportunity employer. 